\title{
Two-Dimensional Spatiotemporal Coding of Linear Acceleration in Vestibular Nuclei Neurons
}

\author{
Dora E. Angelaki, ${ }^{1}$ Geoffrey A. Bush, ${ }^{1, a}$ and Adrian A. Perachio ${ }^{1,2,3}$ \\ Departments of ${ }^{1}$ Otolaryngology, ${ }^{2}$ Physiology and Biophysics, and ${ }^{3}$ Anatomy and Neuroscience, University of Texas \\ Medical Branch, Galveston, Texas 77555
}

Response properties of vertical (VC) and horizontal (HC) canal/otolith-convergent vestibular nuclei neurons were studied in decerebrate rats during stimulation with sinusoidal linear accelerations $(0.2-1.4 \mathrm{~Hz})$ along different directions in the head horizontal plane. A novel characteristic of the majority of tested neurons was the nonzero response often elicited during stimulation along the "null" direction (i.e., the direction perpendicular to the maximum sensitivity vector, $\left.S_{\max }\right)$. The tuning ratio $\left(S_{\min }\right.$ gain $/ S_{\max }$ gain), a measure of the two-dimensional spatial sensitivity, depended on stimulus frequency. For most vestibular nuclei neurons, the tuning ratio was small at the lowest stimulus frequencies and progressively increased with frequency. Specifically, HC neurons were characterized by a flat $S_{\max }$ gain and an approximately 10 -fold increase of $S_{\min }$ gain per frequency decade. Thus, these neurons encode linear acceleration when stimulated along their maximum sensitivity direction, and the rate of change of linear acceleration (jerk) when stimulated along their minimum sensitivity direction. While the $S_{\max }$ vectors were distributed throughout the horizontal plane, the $S_{\min }$ vectors were concentrated mainly ipsilaterally with respect to head acceleration and clustered around the nasooccipital head axis. The properties of VC neurons were distinctly different from those of $\mathrm{HC}$ cells. The majority of VC cells showed decreasing $S_{\max }$ gains and small, relatively flat, $S_{\min }$ gains as a function of frequency. The $S_{\max }$ vectors were distributed ipsilaterally relative to the induced (apparent) head tilt. In type I anterior or posterior VC neurons, $S_{\max }$ vectors were clustered around the projection of the respective ipsilateral canal plane onto the horizontal head plane. These distinct spatial and temporal properties of HC and VC neurons during linear acceleration are compatible with the spatiotemporal organization of the horizontal and the vertical/torsional ocular responses, respectively, elicited in the rat during linear translation in the horizontal head plane. In addition, the data suggest a spatially and temporally specific and selective otolith/canal convergence. We propose that the central otolith system is organized in canal coordinates such that there is a close alignment between the plane of angular acceleration (canal) sensitivity and the plane of lin-

\footnotetext{
Received May 7, 1992; revised Aug. 10, 1992; accepted Sept. 28, 1992

This work was supported by the following grants: NASA NGT 50581, NASA NGT 50165, NASA NAG 2-26, and NIH DC-00385.

Correspondence should be addressed to Dora Angelaki, Department of Neurology, University of Zürich, Frauenklinikstrasse 26, CH-8091 Zurich, Switzerland.

Present address: Laboratory of Sensorimotor Research, National Eye Institute, NIH Building 10, Room 10C101,Bethesda, MD 20892.

Copyright (c) 1993 Society for Neuroscience $0270-6474 / 93 / 131403-15 \$ 05.00 / 0$
}

ear acceleration (otolith) sensitivity in otolith/canal-convergent vestibular nuclei neurons.

[Key words: vestibular, linear acceleration, otolith, spatial tuning, eye movements, vestibulo-ocular reflex, ofolith-ocular reflex, convergence]

The otolith-ocular reflexes are an important part of the vestibulo-ocular reflexes (VOR) that help stabilize visual images on the retina during head movements. Yet, they have not been as well studied as canal-ocular reflexes. Two reasons have impeded our understanding of the otolith-ocular system. First, the organization of these reflexes and the precise mechanism that linear acceleration, encoded by primary otolith afferents (cf. Fernandez and Goldberg, 1976a-c; Goldberg et al., 1990a,b), is processed in order to generate the ocular responses are relatively unknown. Second, different types of otolith-ocular reflexes exist, each having separate roles, different properties, and unique functional utilities (Paige and Tomko, 199 la,b).

Otolith-ocular reflexes can be summarized into three categories: "translational," "tilt," and "off-vertical axis" VOR. Both the translational and tilt VORs can be elicited during stimulation along discrete directions and have been generally called linear vestibulo-ocular reflexes (LVORs). The translational LVORs, compensatory to linear translation, include conjugate horizontal and vertical eye movements during linear translation along the interaural and vertical axes, respectively, and a combination of disconjugate horizontal and vertical eye movements during linear translation along the naso-occipital head axis (Hess and Dieringer, 1991; Paige and Tomko, 1991a,b). The dynamic characteristics of these reflexes have been described as high-pass filters of linear acceleration (Mayne, 1974; Paige and Tomko, 199 la,b). The tilt LVORs are compensatory to head tilt in space (Baarsma and Collewijn, 1975; Hess and Dieringer, 1991; Paige and Tomko, $1991 \mathrm{a}, \mathrm{b})$. The dynamic characteristics of these reflexes have been described as low-pass filters of linear acceleration (Lichtenberg et al., 1982; Hess and Dieringer, 1991; Paige and Tomko, 1991a,b). The third category of otolith-ocular reflexes are compensatory to head rotation (off-vertical axis $V O R$ ). During head rotations about axes tilted from the earth-vertical, the otolith system generates an eye velocity response that is compensatory for head velocity at low frequencies. The static (DC) angular velocity sensitivity of the otolith system is manifested as a steady-state eye velocity during constant velocity off-vertical axis rotation (cf. Correia and Money, 1970; Hess and Dieringer, 1990).

Therefore, based on the oculomotor outputs, the central otolith system is capable of performing as (1) a high-pass filtered linear acceleration detector, (2) a low-pass filtered head position detector, and (3) an angular velocity sensor. Whereas these prop- 
erties of the otolith-oculomotor system have been recognized for some time, the neurophysiological mechanisms underlying the generation of such diverse functions remain unknown. Detailed studies of the static and dynamic properties of primary otolith afferents have indicated that the otolith-ocular reflexes can not be produced by afferent information alone (Fernandez and Goldberg, 1976a-c; Goldberg and Fernandez, 1982), and therefore, additional computations must take placc ccntrally. Significant central otolith processing at the level of the vestibular nuclei has been suggested in the past (Melvill Jones and Milsum, 1969; Schor et al., 1984, 1985).

The anatomical pathways subserving the otolith reflexes also remain largely unknown. The presence of significant otolith/ canal convergence in the vestibular nuclei (Curthoys and Markham, 1971; Searles and Barnes, 1977; Lannou et al., 1980) suggests that the otolith system might not be entirely independent of the canal system. For example, it is possible that the same anatomical pathways comprised of neurons that receive otolith/ canal-convergent inputs might be responsible for the generation of all VORs. That is, horizontal canal (HC)-sensitive neurons projecting to the medial and lateral recti could also exhibit the appropriate otolith sensitivity to generate horizontal eye movements during linear acceleration. Similarly, vertical canal (VC) and otolith sensitivity might cocxist in ncurons participating in the vertical/torsional ocular responses. Thus, otolith/canal convergence might play a crucial role in the investigation of the otolith-ocular system.

Several questions are yet to be answered regarding central otolith processing and otolith/canal convergence. For example, how does the low-pass (necessary for the generation of tilt LVORs) and high-pass (necessary for the translational LVORs) filtering of the linear acceleration input take place? Have these computations already been performed at the level of the vestibular nuclei? Further, is otolith/canal convergence in the vestibular nuclei organized in a spatially and temporally specific manner for the generation of the three categories of spatially and temporally distinct compensatory ocular responses?

In the present study, these questions have been addressed by examining the frequency dependence of the spatiotemporal response properties of otolith/canal-convergent vestibular nuclei neurons in rats during stimulation with pure linear acceleration. Each cell was first characterized in terms of its major canal input and subsequently subjected to a series of linear oscillations at different frequencies and different directions in the horizontal head plane. The goal was to characterize the spatial and temporal properties of these neurons during stimulation with pure linear acceleration and to establish the relationship of these properties to the major canal input of the cell. Based on the specific requirements for the generation of compensatory ocular responses in the vertical/torsional and horizontal VOR pathways during linear acceleration in the horizontal head plane, the following hypotheses were tested. First, otolith/HC-convergent neurons would have high-pass filter characteristics in response to linear acceleration that are appropriate for the generation of the compensatory horizontal translational LVOR. Second, otolith/VCconvergent neurons would exhibit low-pass filter properties, appropriate for the generation of the torsional and vertical tilt LVORs in rats (Hess and Dieringer, 1991). The results demonstrate that $\mathrm{HC}$ - and VC-sensitive vestibular nuclei neurons exhibit distinctly different spatiotemporal properties to linear acceleration. Such different response properties are consistent with the different functional roles of the vertical/torsional and horizontal VOR systems during linear acceleration in the horizontal head plane.

\section{Materlals and Methods}

Surgical procedures. Long Evans rats decerebrated at a precollicular level were used in this study. Details about the surgical procedures have been described in detail elsewhere (Perachio et al., 1992; Bush, 1991). Briefly, the rats were initially anesthetized with a short-lasting anesthetic (Brevital methohexital sodium, $65 \mathrm{mg} / \mathrm{kg}$, i.p.) and decerebrated by ligating the carotid arteries bilaterally and using a combination of aspiration and transection at the rostral pole of the superior colliculus. Access to the vestibular nuclei was accomplished via a craniotomy over the cerebellar cortex. $\mathrm{An} \mathrm{Ag} / \mathrm{AgCl}$ stimulating electrode was implanted adjacent to the oval window in order to characterize the recorded neurons in terms of their response latency to electrical stimulation of the ipsilateral labyrinth. After completion of the surgery, the head was mounted in the stereotaxic head holder with the nose tilted down $26^{\circ}$ (with respect to the stereotaxic horizontal plane) in order to position the horizontal canals approximately parallel to the earth-horizontal plane (Blanks and Torigoe, 1989). In this standard position, yaw rotation of the animal preferentially stimulated the horizontal semicircular canals. A long-lasting paralytic agent, pancuronium bromide (Pavulon), was administered during recordings in order to maintain stability and maximize the isolation time of single neurons. The animal was artificially ventilated and core body temperature was maintained within physiological range $\left(36.5-38^{\circ} \mathrm{C}\right)$. At the end of the recording sessions the animals were given a lethal dose of urethane and perfused with aldehyde fixatives for subsequent histological verification of the recording sites. The completeness of the decerebration was confirmed by histological sections through the level of the transection.

Vestibular stimulation and recording. The animals were placed in a stereotaxic frame, as described above, with the frame being mounted onto a cart that was coupled to a set of linear rails. Sinusoidal linear acceleration of the cart was produced by a servo-controlled DC torque motor. The direction of the cart movement was parallel to the earthhorizontal plane. The stereotaxic frame could be repositioned relative to the direction of travel of the cart, such that linear acceleration along different directions in the horizontal head plane could be delivered. A head-fixed coordinate system was defined in the standard position with the longitudinal axis being the $x$-axis, and the interaural axis being the y-head axis (Fig. 1). Positive directions were forward and out the left ear, respectively. The z-axis was vertical and collinear with gravity when the animal was in the standard position. The direction of the linear acceleration stimulus was specified by its polar angle relative to the positive $\mathrm{x}$-axis in the head-fixed coordinate system (angle $\alpha$ in Fig. 1). Positive polar angles were counterclockwise (CCW), whereas negative polar angles corresponded to clockwise $(\mathrm{CW})$ vector orientations in the head coordinate system.

The sinusoidal stimuli delivered were at one of the following frequencies: $0.2,0.4,0.6,0.8,1.0$, or $1.4 \mathrm{~Hz}$. The peak acceleration was kept constant to $\pm 0.1 \times g\left(g=9.81 \mathrm{~m} / \mathrm{sec}^{2}\right)$ for all frequencies except $0.6 \mathrm{~Hz}$, for which the peak acceleration was $\pm 0.145 \times g$. We had previously tested the sensitivity of vestibular nuclei neurons to different levels of peak linear acceleration ranging from $0.03 \times g$ to $0.145 \times g$ at $0.6 \mathrm{~Hz}$. The response magnitude increased linearly in this amplitude range (A. A. Perachio, unpublished observations). The linear accelerations delivered were outside the frequency and amplitude range of any tilt-sensitive horizontal semicircular canal primary afferent fibers (Perachio et al., 1988). Therefore, an inherent assumption of this study is that the applied linear accelerations activated the otolith receptors exclusively.

Extracellular recordings of single vestibular neurons were made with solution-filled glass micropipettes using standard electrophysiological techniques. Following the completion of each electrode penetration, dye spots were passed at the deepest tract location, allowing subsequent reconstruction of the recording sites. The neurons reported in the present study were located in the medial vestibular nucleus (MVN). Most recordings were made in the left vestibular nuclei. However, some earlier data from $\mathrm{HC}$ cells were collected from the right brainstem. To facilitate inspection of the $\mathrm{HC}$ vector distributions, data from neurons located in the right vestibular nuclei were adjusted by reflecting the vector directions relative to the midline and subsequently treated as having been recorded in the left vestibular nuclei. For example, a right vestibular nuclei vector oriented at $+10^{\circ}$ would be adjusted to an orientation of $-10^{\circ}$. 
Experimental protocol. Upon isolation, each unit was qualitatively identified for its response to angular head acceleration. A series of earthhorizontal and earth-vertical rotations were manually delivered around different head axes (cf. Estes et al., 1975). The neurons were characterized by their major canal input as horizontal canal (HC) or vertical canal (VC) neurons. HC neurons were further classified as type I or type II based on their response polarity during yaw rotation (Duensing and Schaefer, 1959). During most of the experimental sessions, VC neurons were further characterized as type I or type II anterior or posterior canalsensitive cells. Since all VC neurons were recorded in the left vestibular nuclei, they have been characterized as type I or type II left anterior (LA) or left posterior (LP) cells. For example, a type I LA neuron was maximally sensitive during head-vertical rotations in the plane of the left anterior/right posterior canal and was excited by nose down pitch. However, some of the earlier experiments did not permit testing of the neuronal responses during rotations in different vertical head planes. These neurons were only characterized in terms of their response polarity during pitch rotations, and thus could not be identified as type I or type II anterior or posterior canal-sensitive cells.

Following classification of the neurons according to their major canal input, the main stimulus protocol consisting of sinusoidal lincar oscillations at different frequencies was delivered. Each neuron was tested along different head axes (every $15^{\circ}, 30^{\circ}$, or $45^{\circ}$ over a range of $180^{\circ}$ or $360^{\circ}$ ) at one or more of the following frequencies: $0.2,0.4,0.6,0.8,1.0$, and $1.4 \mathrm{~Hz}$. The neural signal was amplified and band-pass filtered $(20$ $\mathrm{Hz}$ to $10 \mathrm{kHz}$ ). The stimulus (position of the cart on the linear track) and neural signals were stored on a tape recorder for subsequent analyses.

Only neurons that received inputs from both the otolith and canal receptor organs were studied in the present investigation. These otolith/ canal-convergent neurons will be referred to by their canal sensitivity classification (i.e., type I/type II HC or VC). It is important to note that all quantitative neuronal response descriptions presented here refer to the properties of these neurons during stimulation with pure linear acceleration. This is a crucial point since, as explaincd below, ncurons exhibited spatiotemporal convergence (STC) characteristics (i.e., different response dynamics along different stimulus directions and nonzero minimum response sensitivity) that have previously only been reported during simultaneous stimulation with both angular and linear acceleration (Baker et al., 1984). Due to limited number of neurons tested at multiple frequencies and orientations, monosynaptic and polysynaptic neurons are presented together in this report.

Data analysis. The neural signals were processed off-line through a time/amplitude window discriminator (BAK Electronics model DIS-1). The discriminator-generated acceptance pulses and the stimulus signal were passed to a peripheral interface device [Cambridge Electronic Design (CED), model 1401] that was connected to and programmed by a microcomputer. The CED functioned as a frequency interrupt device that was programmed to calculate the interspike intervals of the ncuronal responses. Interspike intervals were subsequently converted to instantaneous firing frequency that was averaged into a cycle histogram (256 bins per cycle for each stimulus frequency). In the case of the stimulus signal, the CED performed as an analog-to-digital converter that was programmed to sample the input signal at $100 \mathrm{~Hz}$. The cycle histograms of the response and the stimulus were separately subjected to a linear curve-fitting routine to compute the least-squares fit of the sum of two sinusoids representing the first and second harmonics of the stimulus. Gains were expressed as the ratio of peak firing frequency to peak stimulus intensity (in spikes/sec/g). The phase of the fundamental component of the response was expressed as the difference between peak firing frequency and peak linear acceleration. Only neurons without significant harmonic distortion were included in the analysis (the magnitude of the sccond harmonic component of the response was less than $20 \%$ of the magnitude of the fundamental). Both gain and phase have been expressed relative to linear acceleration, and positive phase values represent phase leads.

Spatiotemporal response properties: $\boldsymbol{S}_{\max }$ and $\boldsymbol{S}_{\min }$ determination. We have previously shown that vestibular nuclei neurons do not necessarily show a null response during stimulation with pure linear acceleration in the horizontal head plane. These cells also exhibit response phases that are a function of stimulus orientation (Angelaki et al., 1992; Bush et al., 1992). Two response vectors (instead of the traditional "polarization vector") are necessary in order to describe quantitatively the response properties of such neurons. Details of the theoretical formulation and its application to vestibular nuclei neuron responses are found elsewhere (Angelaki, 1991a; Angelaki et al., 1992). Briefly, the spatio-

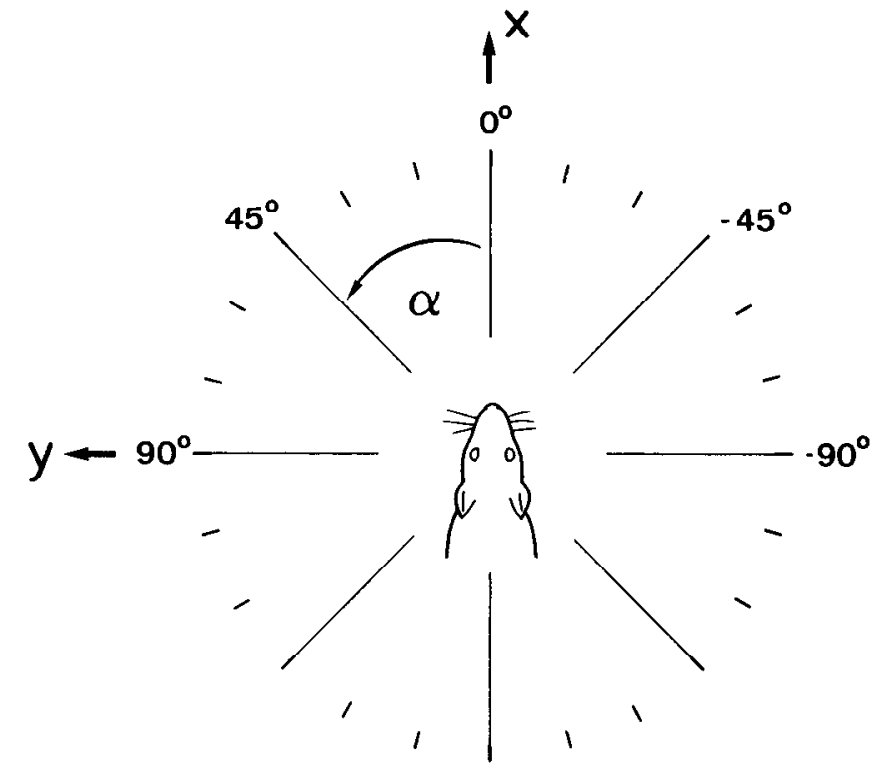

Figure 1. Schematic diagram summarizing the experimental protocol. The direction of each response vector is specified by the polar angle $\alpha$ that the linear acceleration stimulus vector (defined during the forward movement of the cart) forms with the animal's $x$ (naso-occipital)-axis. Positive angles $\alpha$ correspond to $\mathrm{CCW}$ vector orientations in the headfixed coordinate system.

temporal response properties of otolith neurons can be quantitatively described by two vectors that are in spatial and temporal quadrature (i.e., they differ spatially and temporally by $90^{\circ}$ ). The two vectors could be regarded as the semi-major and semi-minor axes of a response ellipse (Angelaki, 1991a). The vector with the largest magnitude $\left(\mathbf{S}_{\max }\right)$ represents the maximum sensitivity, whereas that with the smallest magnitude $\left(\mathbf{S}_{\min }\right)$ is the minimum sensitivity vector of the neuron in the plane of stimulation. In short, such neurons exhibit two-dimensional spatial sensitivity to linear acceleration. A measure of the two-dimensional sensitivity of neurons is the tuning ratio, defined as the gain ratio of the minimum over the maximum sensitivity of the cell. It must be emphasized that the two vectors $\mathbf{S}_{\max }$ and $\mathbf{S}_{\min }$ simply represent "an orthogonal basis" of the two-dimensional spatiotemporal response profiles of neurons and $d o$ not imply convergence of primary otolith afferents with orthogonal polarization vectors (Angelaki, 1991b, 1992b).

A detailed description and statistical comparison between the fits obtained using the two-dimensional treatment and the traditional oncdimensional approach (characterized by a single response vector that result in response gains following the cosine rule and constant phase values at different stimulus orientations) are presented in a different report (G. A. Bush, A. A. Perachio, and D. E. Angelaki, unpublished observations). It has been shown that the two-dimensional treatment presents a more general approach for an accurate quantitative description of vestibular nuclei neuron responses during stimulation with pure linear acceleration. The procedure for the estimation of $\mathbf{S}_{\max }$ and $\mathbf{S}_{\min }$ has been presented in detail elsewhere (Angelaki et al., 1992). Briefly, for each stimulus frequency, four parameters were estimated for the two-dimensional fits (by minimizing the norm of an "error" matrix composed of both the gain and phase values). The four parameters estimated are (1) the gain of the maximum sensitivity vector $\left(\mathbf{S}_{\max }\right),(2)$ its polar angle $(\alpha)$, and (3) its response phase $(\theta)$, as wcll as (4) the gain of the minimum sensitivity vector $\left(\mathbf{S}_{\min }\right)$. For the one-dimensional fits, three parameters were estimated: the gain of $\mathbf{S}_{\max }$, its polar angle, and its response phase.

Figure 2 shows examples of one-dimensional (broken lines) and twodimensional (solid lines) fits of two neuronal response profiles. The example of Figure $2 A$ shows a VC neuron tested at $0.2 \mathrm{~Hz}$ that had a relatively small $\mathbf{S}_{\min }$ gain $(6.2 \mathrm{spikes} / \mathrm{sec} / g)$. A different example, that of an $\mathrm{HC}$ cell tested at $0.6 \mathrm{~Hz}$, is shown on Figure $2 B$. The latter neuron has a large $\mathbf{S}_{\min }$ gain $(70 \mathrm{spikes} / \mathrm{sec} / g)$, as compared to a $\mathbf{S}_{\max }$ gain of $102 \mathrm{spikes} / \mathrm{sec} / \mathrm{g}$. It is clear from visual inspection and from the calculated mean square error values (MSF) that, even in the case of the $\mathrm{VC}$ neuron shown in Figure $2 A$, the two-dimensional fit is more appropriate to fit accurately the gain and phase dependence on stimulus 
A
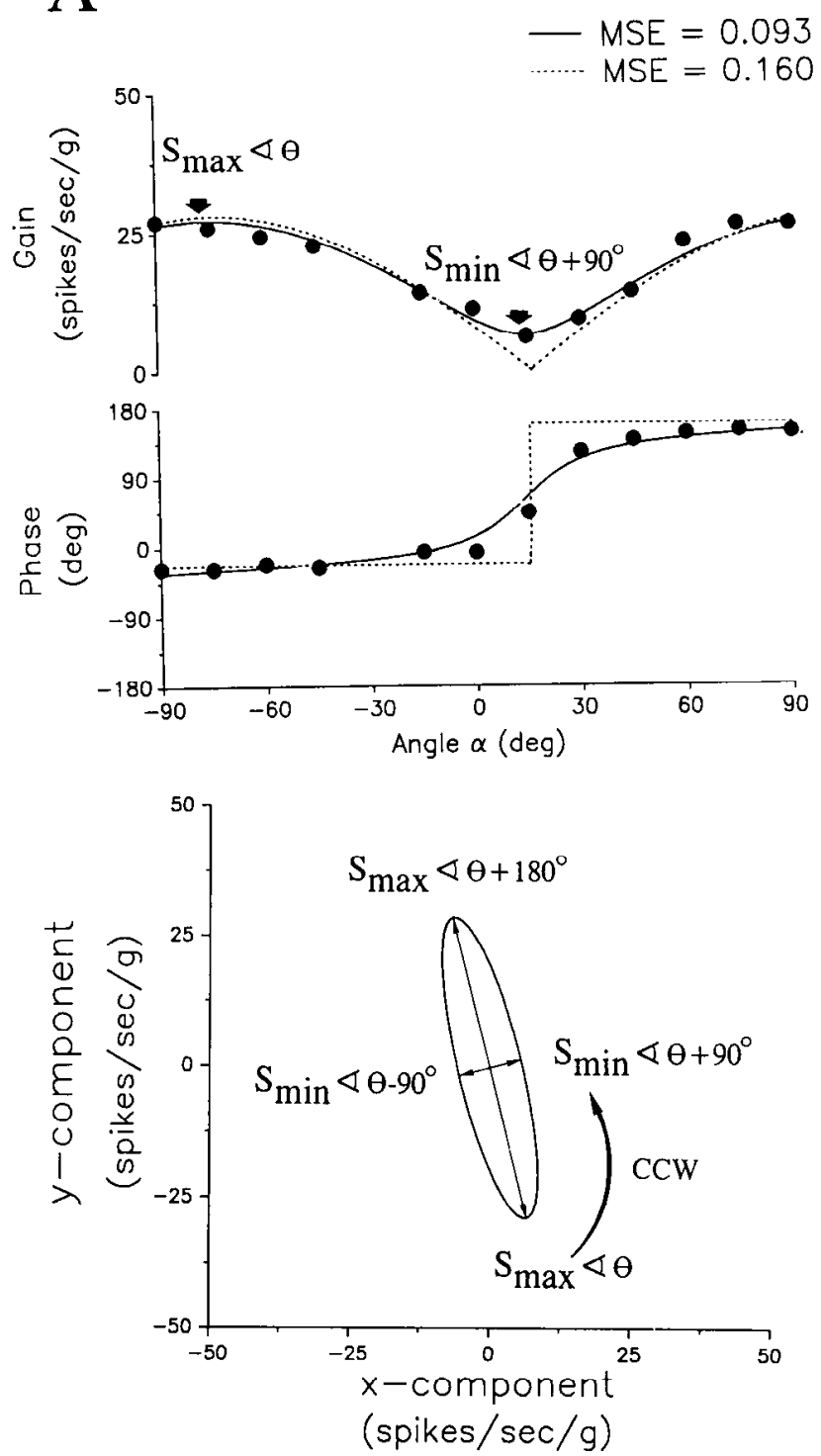

B
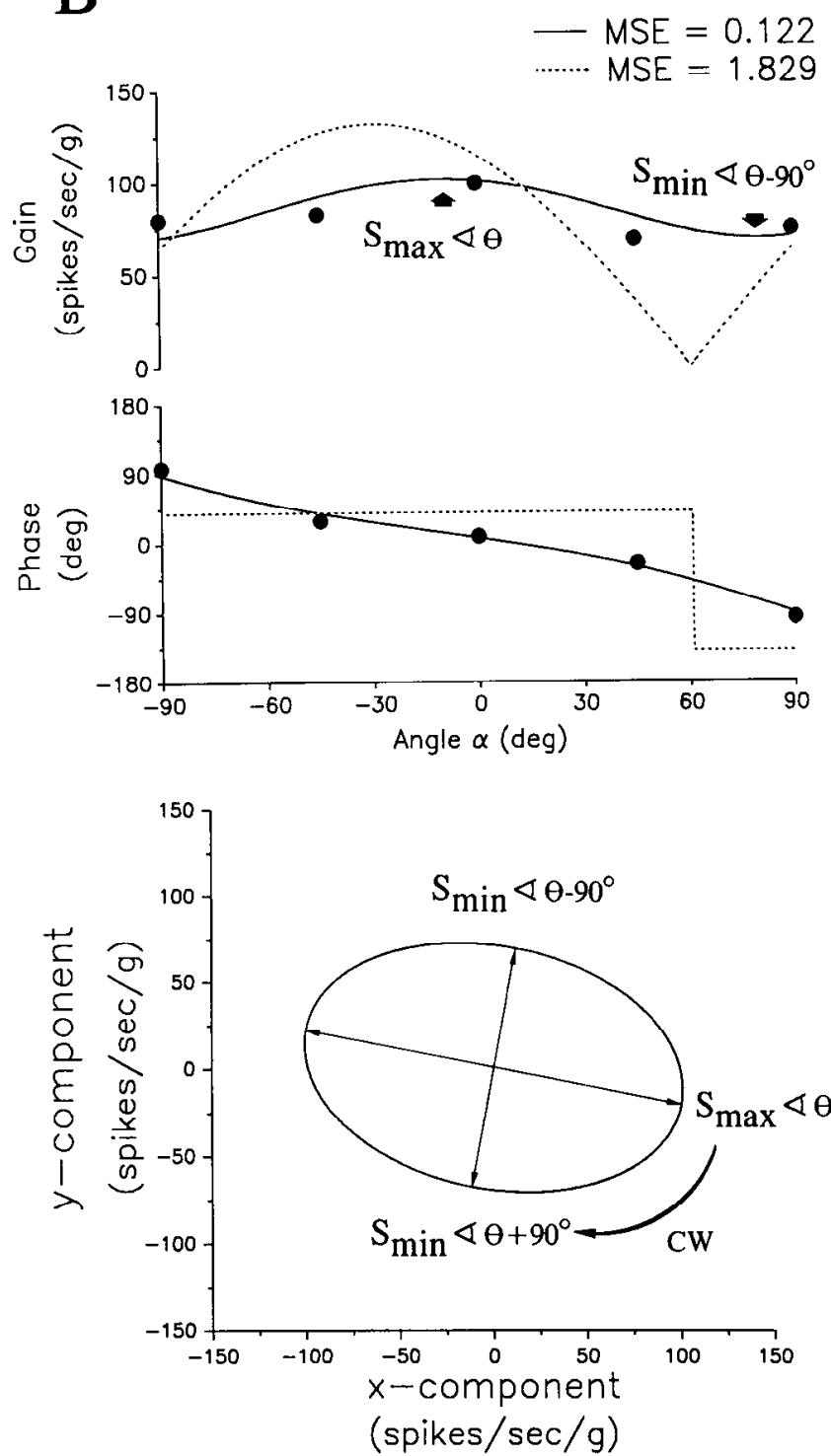

Figure 2. Comparison of the one-dimensional (broken lines) and two-dimensional (solid lines) fits in two neurons. The experimentally determined gain and phase values at different stimulus orientations (represented by their polar angles $\alpha$, Fig. 1) are plotted as solid circles. The corresponding response ellipse for cach ncuron has bcen plotted on the bottom traces. A, A CCW VC neuron that was tested at $0.2 \mathrm{~Hz}$. The parameters cstimated with the two-dimensional treatment are as follows: $\mathbf{S}_{\max }=27$ spikes $/ \mathrm{sec} / g$ at an orientation with $\alpha=-76^{\circ}$ and with a phase of $\theta=-29^{\circ} ; \mathbf{S}_{\min }=$ $6.2 \mathrm{spikes} / \mathrm{sec} / g$. B. A CW HC neuron that was tested at $0.6 \mathrm{~Hz}\left(\mathbf{S}_{\max }=102 \mathrm{spikes} / \mathrm{sec} / g\right.$ at an orientation with $\alpha=-10^{\circ}$ and phase of $\theta=13^{\circ}$; $S_{\min }=70$ spikes $/ \mathrm{sec} / \mathrm{g}$ ). MSE is calculated with the phase expressed in radians and the gain normalized to a maximum value of one for each neuron.

orientation and reveal any potential significance in the presence of a nonzero sensitivity along the "null" direction. Therefore, we have chosen the two-dimensional approach in this analysis of the response properties of vestibular nuclei neurons.

The response phase and orientation of $S_{\min }$ are defined by the spatial and temporal quadrature between $\mathbf{S}_{\max }$ and $\mathbf{S}_{\min }$. From the two possible alternatives (differing by $180^{\circ}$ in either direction or phase), $\mathbf{S}_{\min }$ is further defined as the vector that temporally leads $\mathbf{S}_{\max }$ by $90^{\circ}$. Neurons differ in the relative orientation of their $\mathbf{S}_{\max }$ and $\mathbf{S}_{\min }$ vectors. For example, the neuron shown in Figure $2 A$ has the $\mathbf{S}_{\min }$ vector (defined to lead $\mathbf{S}_{\max }$ by $90^{\circ}$ ) located CCW to $S_{\max }$. This is represented by an increasing phase lead at more positive (counterclockwise) polar angles $\alpha$. For the neuron in Figure $2 B$, the $S_{\min }$ vector is located $\mathrm{CW}$ to $\mathrm{S}_{\max }$. This is represented by a decreasing phase lead (or equivalently, increasing phase lag) at more positive (counterclockwise) polar angles $\alpha$. The relative orientations of $\mathbf{S}_{\max }$ and $\mathbf{S}_{\min }$ are also illustrated in the polar plots of Figure 2, $A$ and $B$. We define counterclockwise (CCW) neurons as those with $\mathbf{S}_{\min }$ vectors located CCW to $\mathbf{S}_{\max }$ (like the VC neuron of Fig. $2 A$ ) and clockwise $(\mathrm{CW})$ neurons as those with $\mathbf{S}_{\min }$ vectors located $\mathrm{CW}$ to $\mathrm{S}_{\max }$ (like the $\mathrm{HC}$ neuron of Fig. $2 B$ ). Only cells with a significant $S_{\min }$ sensitivity could be characterized as $C C W$ or $C W$. Some vestibular nuclei neurons had small tuning ratios $\mathbf{S}_{\min } / \mathbf{S}_{\max }$ (this was particularly true for VC neurons whose stimulation was limited to the lower stimulus frequencies; see Results). Thus, we have set a qualitative criterion that neurons with tuning ratios less than 0.1 at all frequencies tested would not be characterized as $\mathrm{CCW}$ or $\mathrm{CW}$. For such neurons, $\mathbf{S}_{\min }$ was considered practically zero and has not been included in the vector plots (see Figs. $7,10)$.

Transfer function fits. Transfer functions were fitted to the average gain and phase values for $\mathbf{S}_{\max }$ and $\mathbf{S}_{\min }$ of a selected number of $\mathrm{HC}$ and VC neurons. The response gains were normalized to a value of 10 at $0.6 \mathrm{~Hz}$ prior to the calculation of the average. The simplest (i.e., lower order, with fewer parameters) transfer functions that could adequately describe the gain and phase values of $\mathbf{S}_{\max }$ were used for both $\mathrm{VC}$ and $\mathrm{HC}$ neurons. A criterion for acceptance of a certain function as appropriate to describe $\mathbf{S}_{\max }$ was based on the mean square error (MSE) value (calculated based on the normalized gains and on the phase values expressed in radians). After the appropriate function and its parameters 
Table 1. Transfer function fits for $S_{\max }$ and $S_{\min }$ of $\mathrm{HC}$ and VC neurons

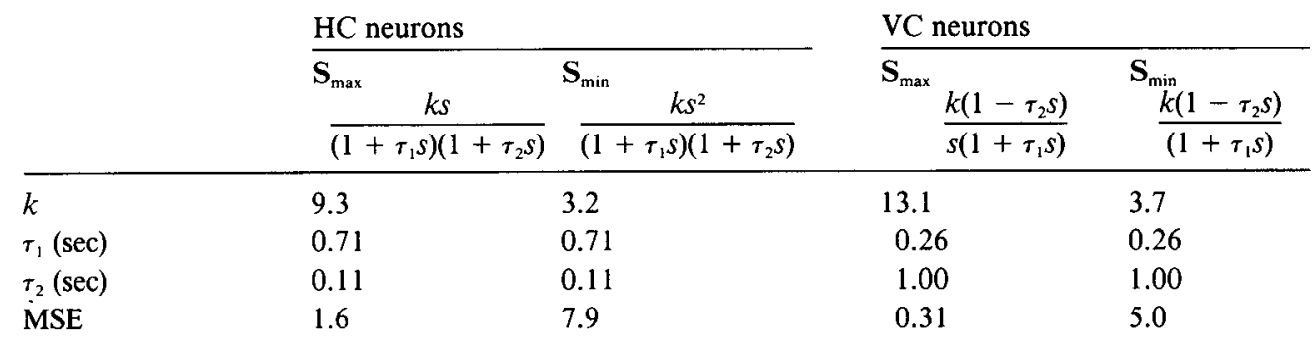

In the above equations, $s=\sigma+j w$ is the complex angular frequency. Multiplication with $s$ in the frequency domain corresponds to a differentiation in the time domain.

werc detcrmincd by fitting the gain and phase values of $\mathbf{S}_{\max }$, all parameters were fixed (except a scalar proportionality constant $k$; Table 1) and the derivative of the transfer function was compared to the gain and phase values of $S_{\min }$. Thus, the derivative of the transfer function of $\mathbf{S}_{\max }$ was fitted with one degree of freedom (the proportionality constant $k$ ) to the gain and phase values of $\mathbf{S}_{\min }$. If $\mathbf{S}_{\min }$ is the derivative of $\mathbf{S}_{\max }$, the gain and phase values of $\mathbf{S}_{\min }$ should be adequately described by such a function.

The procedure described above estimated the parameters of the transfer functions (i.e., the time constants $\tau_{1}$ and $\tau_{2}$; see Table 1) from the $\mathbf{S}_{\max }$ gain and phase values. Similar values for $\tau_{1}$ and $\tau_{2}$ were obtained by first fitting $\mathbf{S}_{\min }$ and subsequently comparing the integral of that transfer function with the $S_{\max }$ gain and phase. We chose to report the results obtained from the former procedure because of the large $\mathbf{S}_{\max }$ responses and the variability in $S_{\min }$ for $V C$ ncurons. It should be cmphasized that the functions used are only descriptive, not directly related to the transfer functions proposed for primary otolith afferents (Fernandez and Goldberg, 1976c; Goldberg et al., 1990a), and do not necessarily suggest any temporal filtering between the otolith afferents and the vestibular nuclei neurons (Angelaki, 1991b).

\section{Results}

A total of $48 \mathrm{VC}$ and $114 \mathrm{HC}$ neurons that received convergent otolith input were isolated in the brainstem of decerebrate rats. A detailed description of the static response properties and the spatiotemporal response profiles during sinusoidal linear acceleration at a single frequency is presented elsewhere (Bush, Perachio, and Angelaki, unpublished observations). This report focuses on the frequency dependence of the spatiotemporal response properties of $\mathrm{HC}$ and VC neurons during stimulation with pure linear acceleration. The neuronal responses to linear acceleration were explored by sinusoidally oscillating the animals at several frequencies along different directions in the horizontal head plane. Figure 3 shows examples of two HC neurons that were tested at different frequencies and orientations. The frequency characteristics of the gain and phase of both $\mathrm{HC}$ neurons shown in Figure 3 were dependent upon stimulus direction. For a quantitative evaluation of the frequency dependence of gain and phase among neurons, the two response vectors $\mathbf{S}_{\max }$ and $\mathbf{S}_{\min }$ were estimated for each cell at each stimulus frequency (see Materials and Methods). Due to the dependence of the response dynamics of neurons on stimulus orientation, cells that were tested at multiple frequencies but at a single orientation have not been included in this study (estimation of the $\mathbf{S}_{\max }$ and $\mathbf{S}_{\min }$ vectors requires knowledge of the neuronal responses along a minimum of two stimulus orientations in the horizontal plane; see Angelaki et al., 1992).

There were three observations common to both $\mathrm{HC}$ and $\mathrm{VC}$ neurons. First, the direction of $\mathbf{S}_{\max }$ and $\mathbf{S}_{\min }$ did not change with frequency. This is illustrated in the top plots of Figure 4 , where the $\mathbf{S}_{\max }$ vector orientations of $7 \mathrm{HC}$ (left) and $11 \mathrm{VC}$ neurons (right) have been plotted as a function of stimulus frequency.
Second, the characterization of a neuron as CW or CCW was frequency independent; that is, the phase of $S_{\min }$ relative to $S_{\max }$ did not change from $90^{\circ}$ lead to $90^{\circ}$ lag or vice versa. Equivalently, a neuron whose phase lead increased (decreased) at more positive (CCW) stimulus orientations exhibited the same phase dependence at all frequencies. This observation, of course, could be made only for frequencies at which the two-dimensional sensitivity of the cell was significant (tuning ratio larger than 0.1 ; see Materials and Methods). When the tuning ratio of the neuron (defined as the gain ratio $\mathbf{S}_{\min } / \mathbf{S}_{\max }$ ) was small, phase was independent of stimulus orientation and no $\mathrm{CCW} / \mathrm{CW}$ classification could be made.

The third similarity between $\mathrm{HC}$ and VC neurons concerned the frequency dependence of the two-dimensional sensitivity. For the majority of $\mathrm{HC}$ and $\mathrm{VC}$ vestibular nuclei neurons, the tuning ratio increased as the frequency of stimulation increased. The frequency dependence of tuning ratio in $\mathrm{HC}$ and $\mathrm{VC}$ neurons has been plotted on the bottom panels of Figure 4. An increasing tuning ratio as a function of frequency suggests that neurons, which had narrowly tuned profiles (i.e., they were characterized by relatively flat phase and a cosine-shaped gain vs stimulus orientation) at low frequencies, showed a more broadly tuned response profile during stimulation at higher frequencies. Despite the similarities in the orientation and gain ratio of the two response vectors, the temporal dependence of $\mathbf{S}_{\max }$ and $\mathbf{S}_{\min }$ was distinctly different between the $\mathrm{HC}$ and VC cells. The temporal and spatial properties of $\mathrm{HC}$ and $\mathrm{VC}$ neurons will be described separately below.

\section{HC neurons}

A total of 33 type I and 22 type II HC neurons were tested during sinusoidal linear acceleration along multiple directions. The mean $S_{\max }$ and $S_{\min }$ gains were, respectively, $44.3 \pm 20.8$ $( \pm \mathrm{SD})$ spikes $/ \mathrm{sec} / g$ and $10.9 \pm 8.1$ spikes $/ \mathrm{sec} / g(n=16 ; 0.2$ Hz) and $75.8 \pm 73.2 \mathrm{spikes} / \mathrm{sec} / g$ and $18.2 \pm 20.8 \mathrm{spikes} / \mathrm{sec} /$ $g(n=46 ; 0.6 \mathrm{~Hz})$. The $\mathbf{S}_{\min }$ gain values for both frequencies were statistically different from zero $(p<0.05)$. Seven of these neurons, which were subsequently histologically identified as being located in the MVN, were successfully tested at multiple frequencies and orientations. The frequency dependence of $\mathbf{S}_{\max }$ and $\mathbf{S}_{\min }$ for these seven $\mathrm{HC}$ neurons (four type $\mathrm{I}$ and three type II HC cells) is shown in Figure 5. Among these HC cells, four neurons were $\mathrm{CCW}$ and three were $\mathrm{CW}$ (see Materials and Methods). For six of the seven neurons, the $\mathbf{S}_{\max }$ gain remained approximately constant $(0.2 \mathrm{~Hz}, 32.4 \pm 14.5 ; 1 \mathrm{~Hz}, 37.0 \pm 16.9)$ whereas the $\mathbf{S}_{\min }$ gain showed a significant increase as the frequency of stimulation increased $(0.2 \mathrm{~Hz}, 3.4 \pm 1.8 ; 1 \mathrm{~Hz}, 13.6$ \pm 8.7 ). Thus, these $\mathrm{HC}$ neurons had maximum sensitivity vec- 

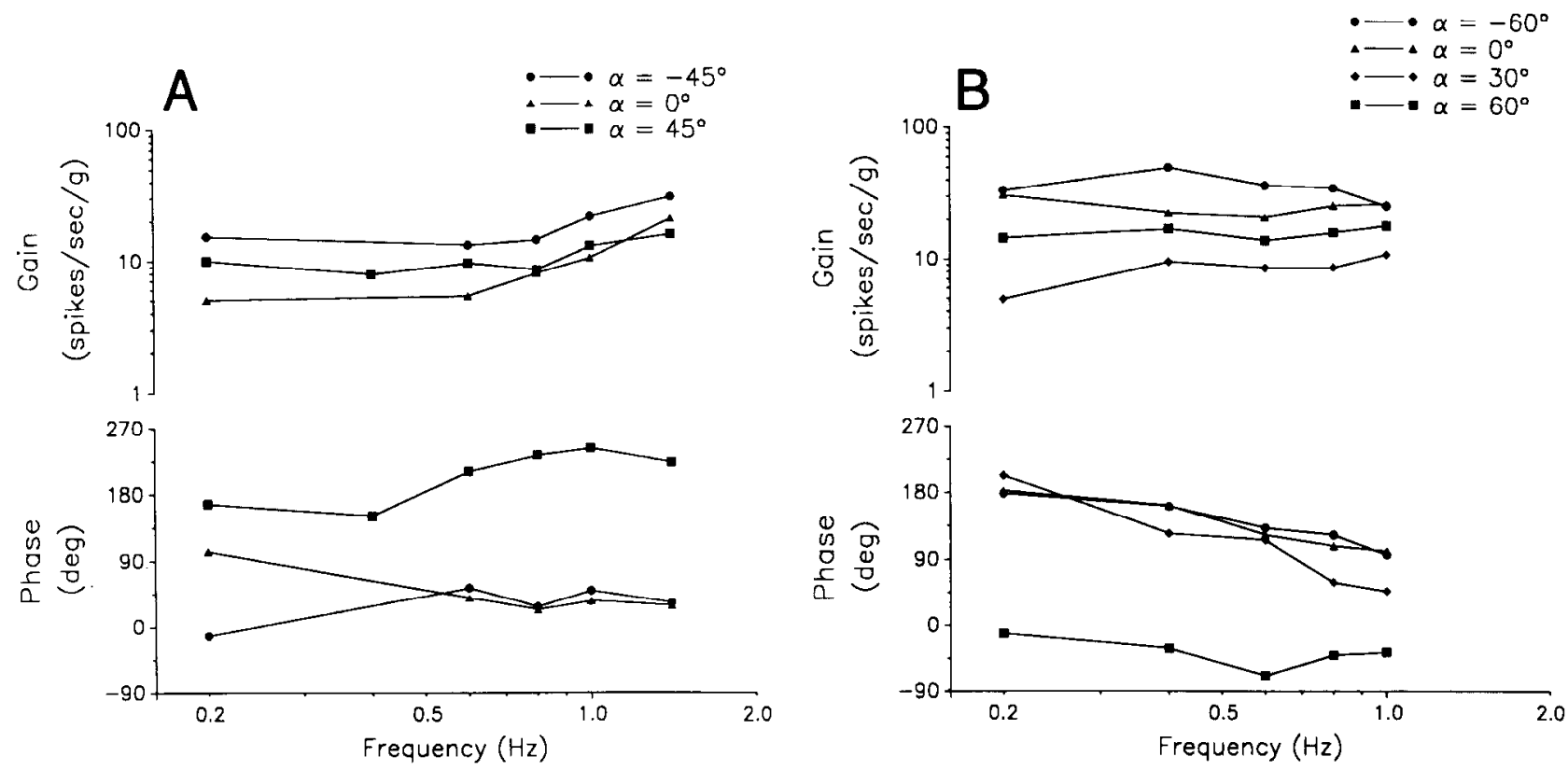

Figure 3. Examples of the frequency response of two HC neurons (type I) during stimulation along different head orientations specified by the polar angle $\alpha$ (Fig. 1). Both gain and phase have been expressed relative to forward linear acceleration. $A$, The neuron's $\mathbf{S}_{\max }$ vector was estimated to be at $\alpha=-72^{\circ}$. $B$, The neuron's $S_{\max }$ vector was estimated to be at $\alpha=127^{\circ}$.

tors that reliably encode linear acceleration (flat gain and nearly zero phase or relatively small phase leads in the mid-frequency range). In addition, when stimulated along their minimum sensitivity vector, these $\mathrm{HC}$ neurons respond in proportion to the derivative of linear acceleration (jerk). One type I neuron located in the MVN (identified with the asterisk in Fig. 5 and with broken lines in the tuning ratio plots of Fig. 4) exhibited the opposite gain dependence of $\mathbf{S}_{\max }$ and $\mathbf{S}_{\min }$ on frequency: $\mathbf{S}_{\max }$ gain increased, whereas $\mathbf{S}_{\min }$ gain was flat. Thus, this neuron exhibited the largest two-dimensional sensitivity at the lowest frequencies.

The average gain (normalized to a value of 10 at $0.6 \mathrm{~Hz}$ ) and
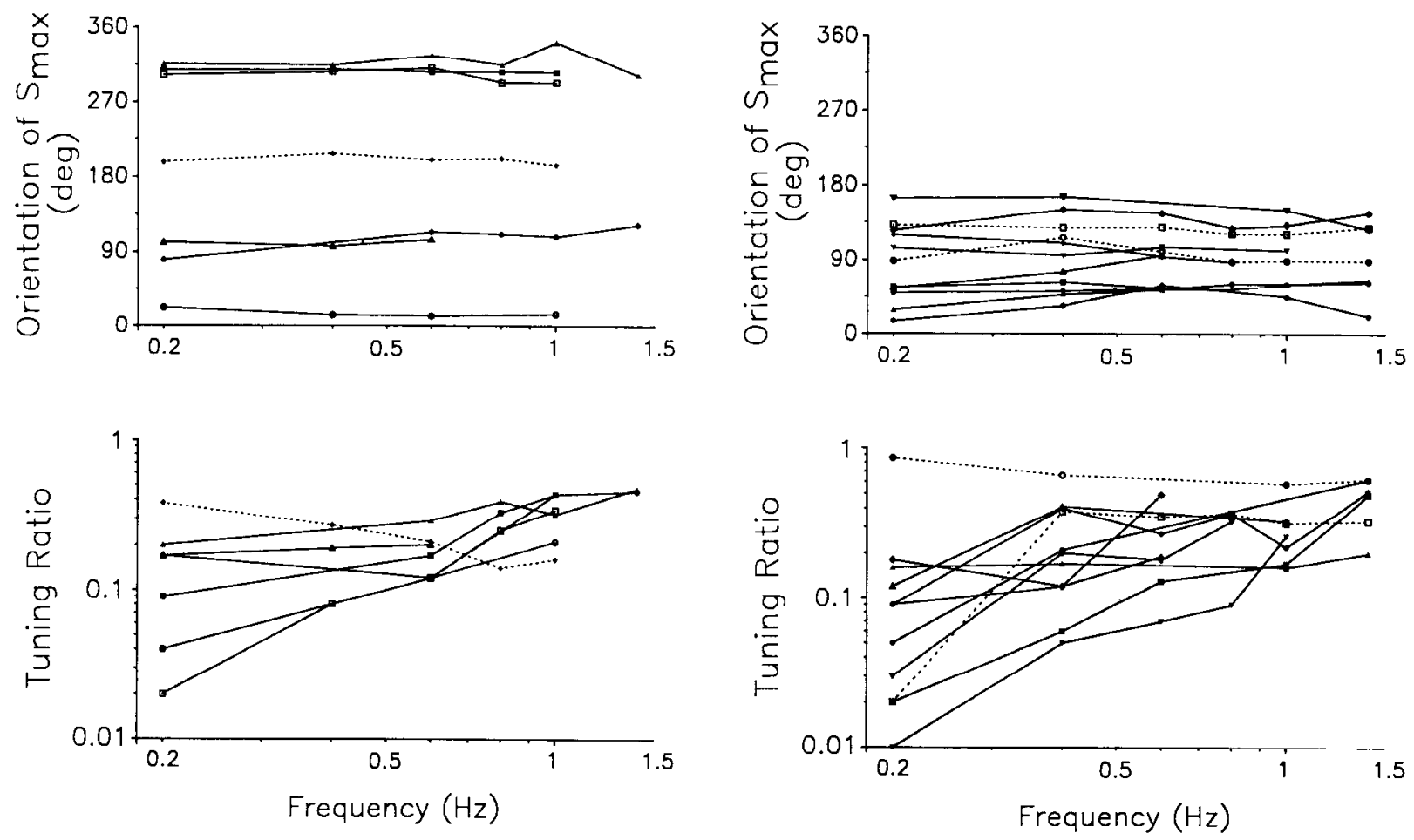

Figure 4. Frequency dependence of the $\mathbf{S}_{\max }$ vector orientation (top) and tuning ratio (bottom). Seven HC neurons (left) and 11 VC neurons (right) are plotted. Broken lines are used for one HC and two VC neurons (marked with asterisks in Figs. 5 and 8 , respectively) whose frequency dependence was different from the rest of the cells. 

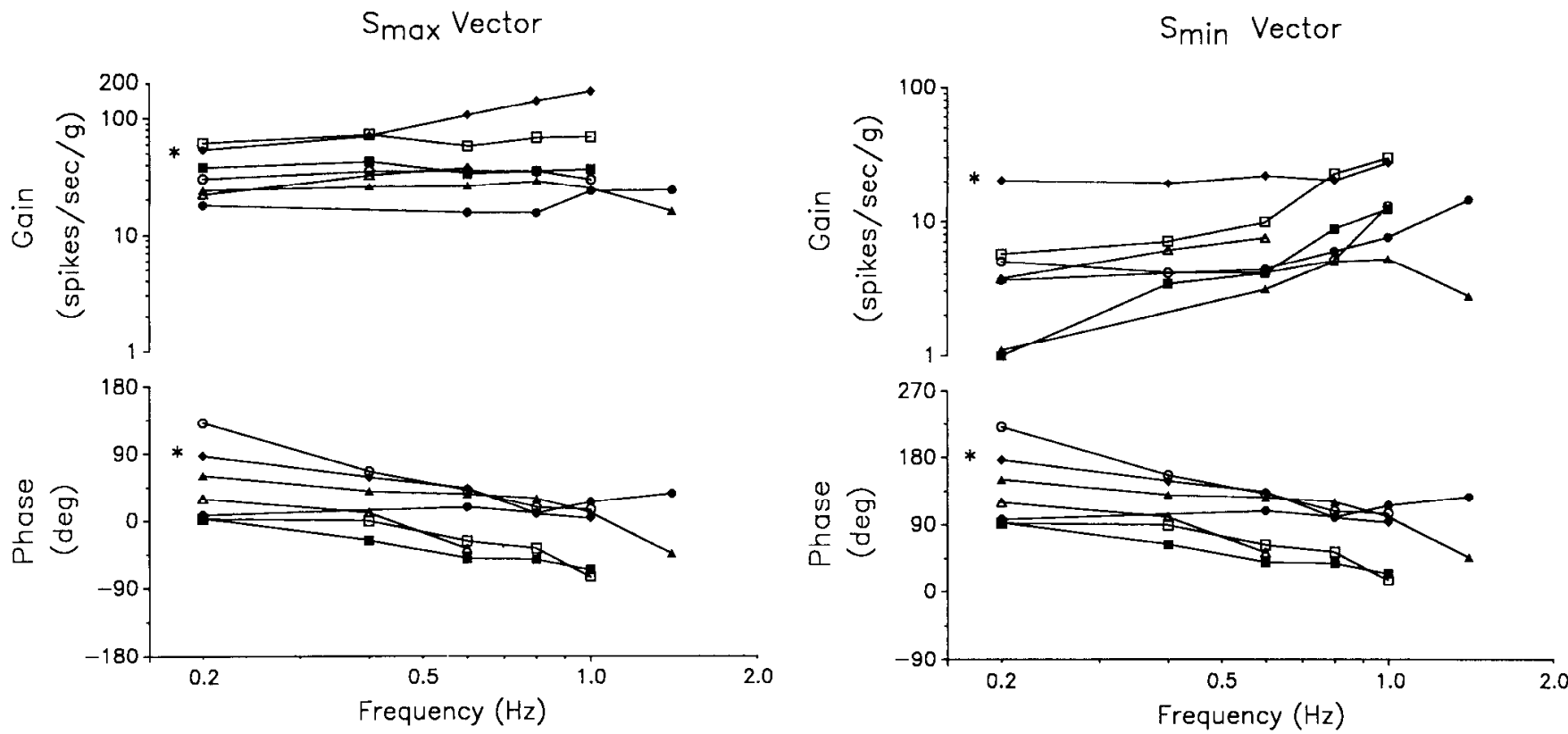

Figure 5. $\mathrm{HC}$ neurons. The gain and phase values of $\mathrm{S}_{\max }$ and $\mathbf{S}_{\min }$ for $\mathrm{HC}$ neurons are plotted as a function of frequency. The asterisks indicate the gain and phase of one type I HC neuron whose properties were opposite from the other six cells. Zero phase indicates neuronal responses in phase with linear acceleration.

phase for the six neurons whose response gain and phase were plotted in Figure 5 are presented in Figure 6. The relatively flat $\mathbf{S}_{\max }$ and the approximately unity slope of increase in $\mathbf{S}_{\min }$, as well as the constant phase difference of $90^{\circ}$ at all frequencies, suggests that there is a derivative relationship between the two response vectors. To quantify this observation, two functions, one of which is the time derivative of the other (Table 1), were fitted to the average $\mathbf{S}_{\max }$ and $\mathbf{S}_{\min }$ values (Fig. 6 , solid lines). As explained in Materials and Methods, the procedure for the fitting was as follows. First, the lowest-order function that adequately described the frequency dependence of $\mathbf{S}_{\max }$ was fitted to its gain and phase values. Subsequently, the derivative of this function was compared to the gain and phase values estimated for $\mathbf{S}_{\min }$. In the latter step, the time constants were restrained to the values estimated for $\mathbf{S}_{\max }$ and the only parameter allowed to vary was the proportionality constant $k$ (Table 1). The MSE values gave a quantitative measure for the goodness of fit.

The distributions of the maximum and minimum sensitivity vector directions in response to linear acceleration in the horizontal head plane were examined for possible clustering around specific head axes. Cells whose response vectors were estimated at multiple frequencies are represented by their mean direction
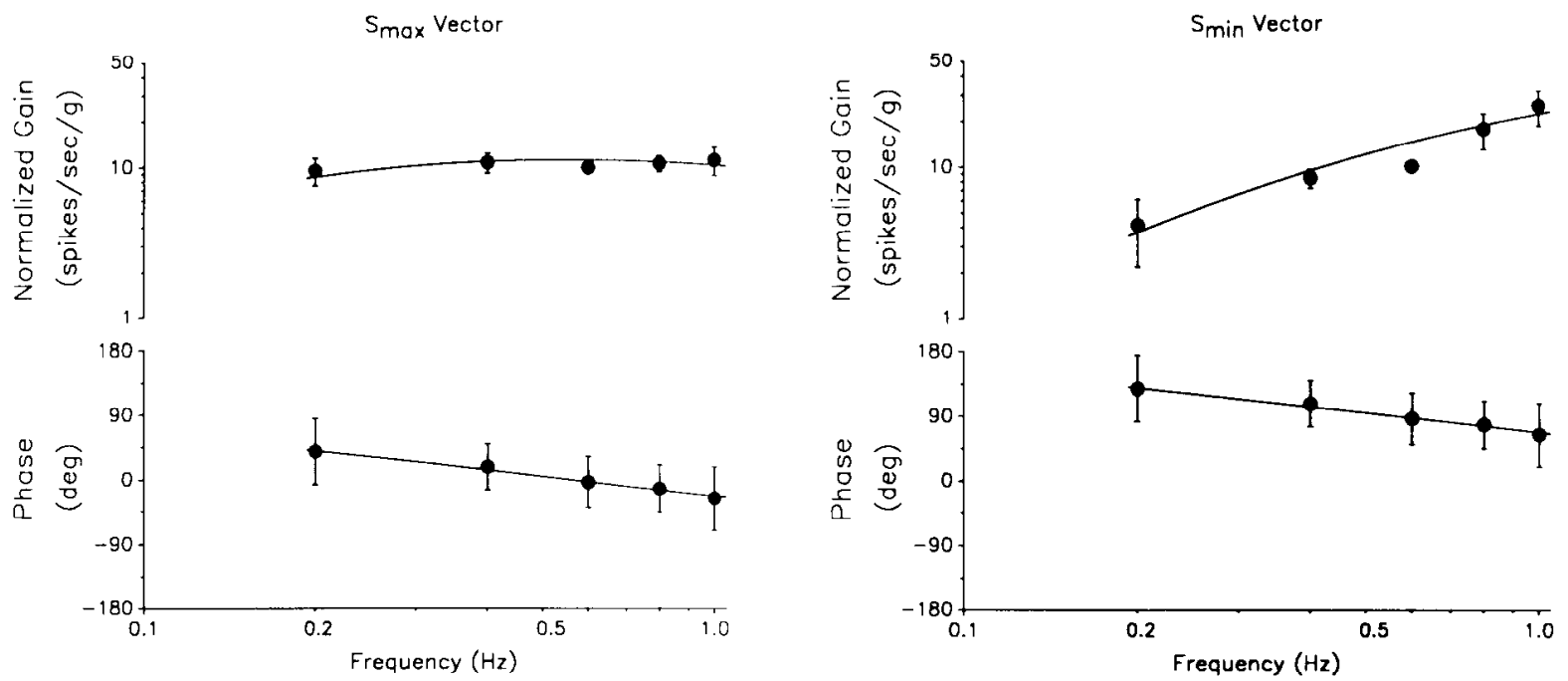

Figure 6. HC neurons. The average gain and phase values estimated for $\mathbf{S}_{\max }$ and $\mathbf{S}_{\min }$ (solid circles) have been plotted as a function of frequency. For each neuron, the gains have been normalized to a value of 10 at $0.6 \mathrm{~Hz}$ before the averages were calculated. Both gain and phase have been expressed relative to linear acceleration. Error bars represent SDs (six neurons). The superimposed solid lines are the fits of the transfer functions presented in Table 1. 
A

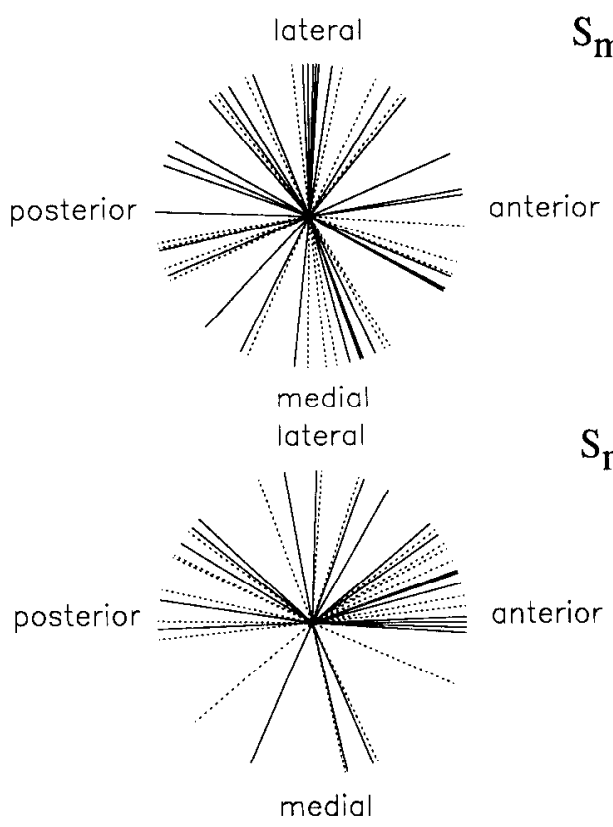

B
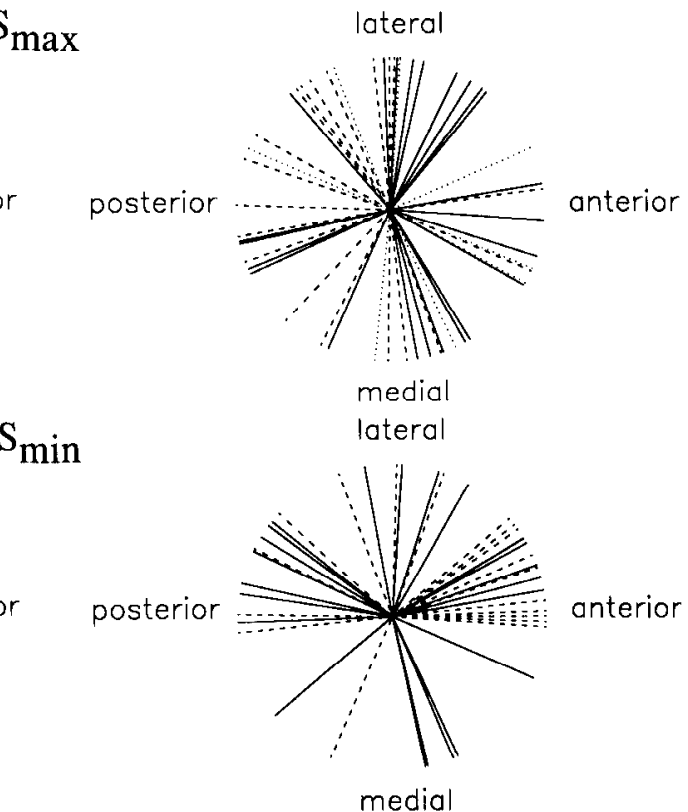

Figure 7. Neural response vector distributions for $\mathrm{HC}$ neurons (top, $\mathrm{S}_{\max }$; hottom,, $\mathbf{S}_{\mathrm{min}}$ ). $A$, Solid lines are used for type I HC neurons $(n=33)$ and broken lines are used for type II $\mathrm{HC}$ neurons $(n=22) . B$. Solid lines are used for $\mathrm{CCW} \mathrm{HC}$ neurons, dashed lines are used for $\mathrm{CW} \mathrm{HC}$ neurons, and dotted lines designate narrowly tuned cells. The response vectors have been expressed relative to linear acceleration. Cells tested at multiple frequencies are represented by their average direction. The vectors plotted have equal lengths and do not depict sensitivity differences among neurons. averaged across all frequencies. Because the magnitudes of the $\mathbf{S}_{\max }$ and $\mathbf{S}_{\min }$ vectors are frequency dependent, all vectors have been normalized to a sensitivity of one. The directions of $\mathbf{S}_{\max }$ vectors (relative to head acceleration) have been plotted in Figure 7 (top). The distribution of the response vectors for type I $(n=33$; solid lines, Fig. $7 A)$ and type II $(n=22$; broken lines, Fig. 7A) were similar. For both type I and type II HC neurons, the $\mathbf{S}_{\max }$ vectors were found to be distributed throughout the horizontal head plane. The vector distributions have also been plotted in Figure $7 B$ for CCW (solid lines), CW (dashed lines), and narrowly tuncd (dotted lines) cells. Since the maximum sensitivity vector was not determined under static conditions, the vectors shown in Figure 7 do not necessarily correspond to the response polarity during static tilts. Rather, for neurons that were tested at multiple frequencies, the $\mathbf{S}_{\max }$ vectors depict the response polarity at $0.6 \mathrm{~Hz}$, where the majority of $\mathrm{HC}$ neurons were tested.

The minimum sensitivity vectors of 24 of 33 type I and 21 of 22 type II $\mathrm{HC}$ neurons are plotted on the bottom panel of Figure 7 (the remaining 10 neurons had tuning ratios less than 0.1 ). The distribution of $\mathbf{S}_{\min }$ vectors (bottom) is different from that of $\mathbf{S}_{\max }$ (top). There was a clear direction predominance of $\mathbf{S}_{\min }$ vectors in the lateral half(ipsilateral) of the horizontal plane. The clustering of $\mathbf{S}_{\min }$ vectors between $0^{\circ}$ and $45^{\circ}$ and between $135^{\circ}$ and $180^{\circ}$ is apparent. The observation that the distributions of $\mathbf{S}_{\max }$ and $\mathbf{S}_{\min }$ are not identical (i.e., simply rotated by $90^{\circ}$ ) might seem surprising at first. This difference in the orientations of $\mathbf{S}_{\max }$ and $\mathbf{S}_{\min }$ vectors is present because of the two possible relative orientations of the two vectors. For approximately half of the $\mathrm{HC}$ neurons (shown with dashed lines in Fig. $7 B$ ), the $\mathbf{S}_{\min }$ vector was located $C W$ relative to the $S_{\max }$ vector. For the remaining cells (shown with solid lines in Fig. $7 B$ ), the $\mathbf{S}_{\min }$ vector was CCW relative to the $\mathbf{S}_{\max }$ vector.

\section{$V C$ neurons}

A total of $25 \mathrm{VC}$ neurons were examined during sinusoidal linear acceleration along multiple stimulus directions in the horizontal head plane. The mean $\mathbf{S}_{\max }$ and $\mathbf{S}_{\min }$ gains were $64.4 \pm 72.4$ spikes $/ \mathrm{sec} / g$ and $3.8 \pm 5.6$ spikes $/ \mathrm{sec} / g(n=22 ; 0.2 \mathrm{~Hz})$ and $42.6 \pm 48.9 \mathrm{spikes} / \mathrm{sec} / g$ and $12.6 \pm 17.7 \mathrm{spikes} / \mathrm{sec} / g(n=9$; $0.6 \mathrm{~Hz}$ ), respectively. The $S_{\min }$ values at $0.2 \mathrm{~Hz}$, but not at 0.6 $\mathrm{Hz}$, were statistically different from zero $(p<0.05)$. Further, comparison of the $\mathbf{S}_{\min }$ sensitivities between $\mathrm{HC}$ and VC neurons demonstrated that VC cells had significantly smaller minimum sensitivity gains compared to HC neurons $(t=2.9, \mathrm{df}=36, p$ $<0.05$ at $0.2 \mathrm{~Hz}$ ). The frequency dependencies of $\mathbf{S}_{\max }$ and $\mathbf{S}_{\min }$ gain and phase have been plotted in Figure 8. Most ( 9 of 11) neurons that were successfully tested at multiple frequencies exhibited a consistent decrease in their maximum sensitivity gain as the stimulus frequency increased. For these nine neurons that showed a decrease in gain as a function of stimulus frequency, the mean $\mathbf{S}_{\max }$ gain dropped from $53.5 \pm 33.8$ spikes/ $\mathrm{sec} / g$ at $0.2 \mathrm{~Hz}$ to $15.9 \pm 11.7 \mathrm{spikes} / \mathrm{sec} / g$ at $1 \mathrm{~Hz}$. In contrast, the $\mathbf{S}_{\min }$ values were small and variable, with no consistent trend among neurons. Two neurons (shown with asterisks in Fig. 8 and with broken lines in the tuning ratio plots of Fig. 4) did not follow this response pattern; these $\mathrm{VC}$ neurons were characterized by approximately $90^{\circ}$ phase leads compared to the rest of the population and increasing gains for both $\mathbf{S}_{\max }$ and $\mathbf{S}_{\min }$ at higher stimulus frequencies. Based on the histological reconstructions of the recording sites, these neurons were also located in the MVN. Due to the limited number of neurons with this response pattern, they were not further analyzed.

Even though the minimum sensitivity gains of VC ncurons were often small and variable, it would be interesting to assess whether the derivative relationship between $\mathbf{S}_{\max }$ and $\mathbf{S}_{\min }$ is not only confined to HC neurons. Inspection of Figure 8 with the approximately 10 -fold decrease of $S_{\max }$ gain per decade of frequency and the relatively flat $\mathbf{S}_{\min }$ gain values across frequency suggests that a derivative relationship might also describe the temporal characteristics of $\mathbf{S}_{\max }$ and $\mathbf{S}_{\min }$ response vectors in $\mathrm{VC}$ cells. Thus, VC neurons that showed a decrease in the $\mathbf{S}_{\max }$ gain were examined as to whether $\mathbf{S}_{\text {min }}$ could also be considered as proportional to the time derivative of $\mathbf{S}_{\max }$. Two functions, one 

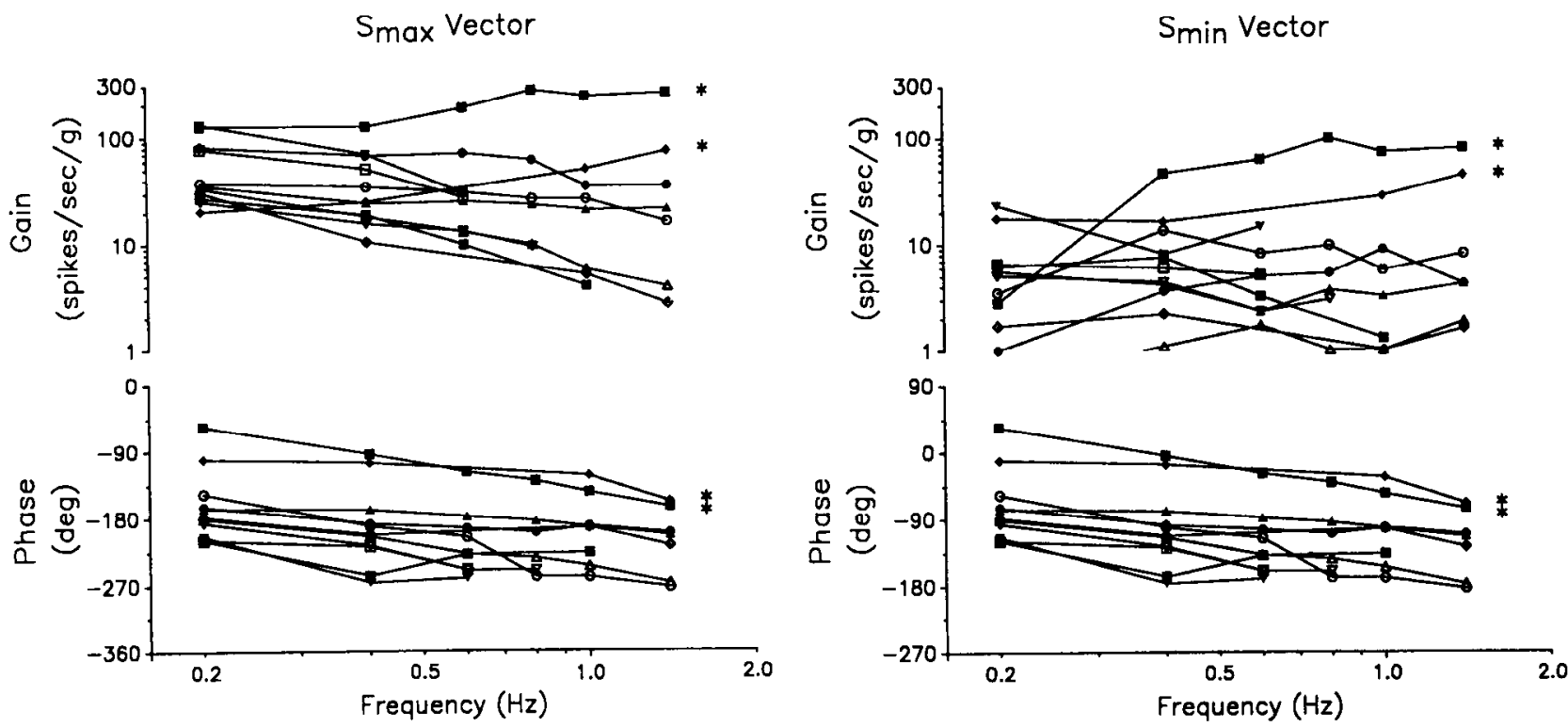

Figure 8. VC neurons. The gain and phase values of $\mathbf{S}_{\max }$ and $\mathbf{S}_{\min }$ of $11 \mathrm{VC}$ neurons are plotted as a function of frequency. The asterisks indicate the gain and phase response of two VC neurons whose properties differed from the other nine neurons. Zero phase indicates neuronal responses in phase with linear acceleration.

of which is the time derivative of the other (Table 1), were fitted to the average $\mathbf{S}_{\max }$ and $\mathbf{S}_{\min }$ gain and phase values of $\mathrm{VC}$ cells. The average normalized gain and phase (solid circles) and the fitted curves (solid lines) are presented in Figure 9. Only four neurons whose responses were tested at all six frequencies were included in the averages. Despite the large variability in $\mathbf{S}_{\min }$, a derivative relationship between $\mathbf{S}_{\min }$ and $\mathbf{S}_{\max }$ provided a good fit to the data (Table 1).

Unlike the $\mathbf{S}_{\max }$ vector distribution of $\mathrm{HC}$ neurons that was broad and covered the whole horizontal plane (Fig. 7), the maximum sensitivity vectors of VC neurons were primarily directed unilaterally. In addition, there was a paucity of vectors along the naso-occipital axis (anterior-posterior direction). The $\mathbf{S}_{\max }$ vectors of VC neurons are plotted in Figure 10. Solid lines are used for type I LA neurons, dashed lines are used for type I LP neurons, and dotted lines demonstrate unidentified cells (see Materials and Methods). It must be pointed out that the vector distributions of Figure 10 represent the response polarity at the lowest tested frequency. In addition, the response vectors of $\mathrm{VC}$ neurons in Figure 10 have been plotted relative to head position and not head acceleration (notice that phase relative to linear acceleration is centered around $-180^{\circ}$ in Figs. 8, 9). This was
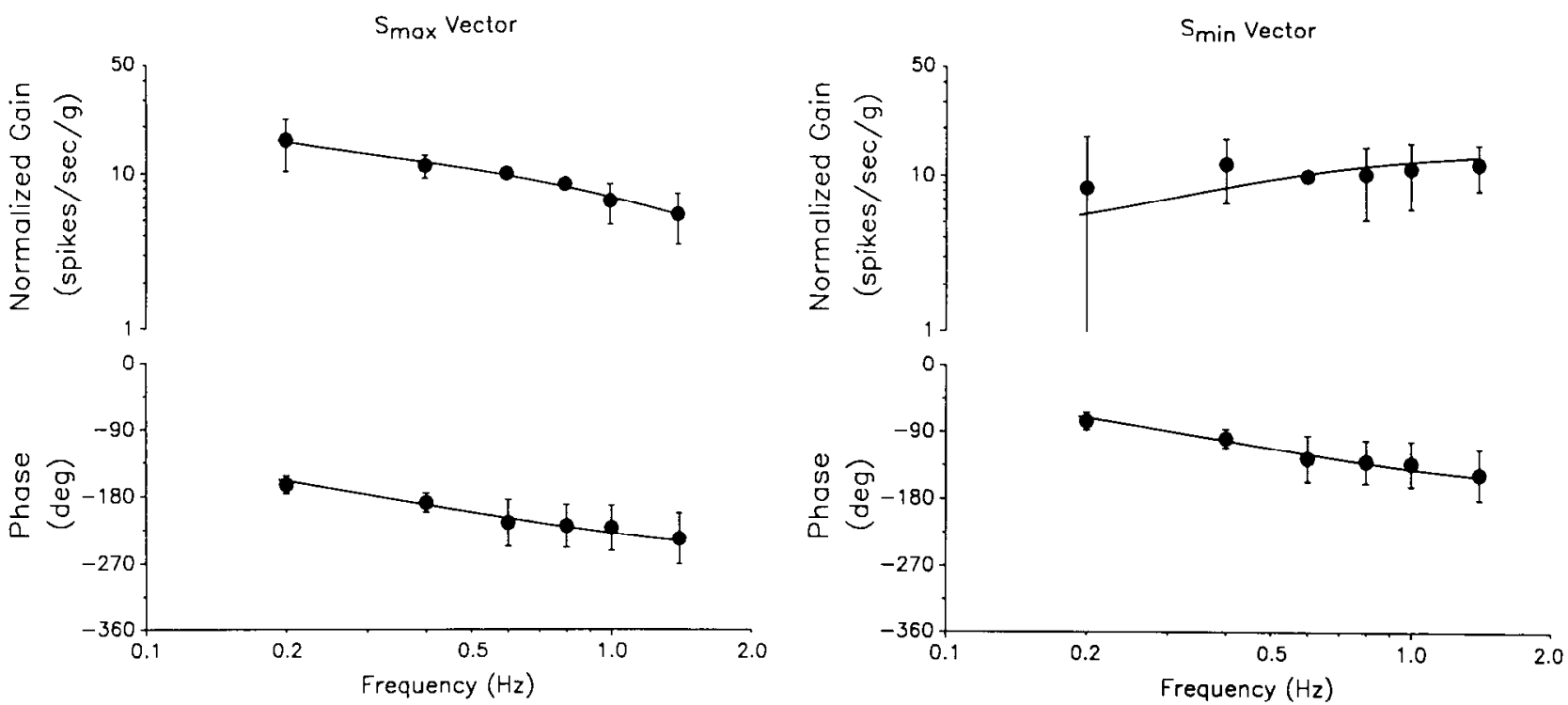

Figure 9. VC neurons. The average gain and phase values estimated for $\mathbf{S}_{\max }$ and $\mathbf{S}_{\min }$ (solid circles) have been plotted as a function of frequency. Error bars represent SDs (four neurons). For each neuron, the gains have been normalized to a value of 10 at $0.6 \mathrm{~Hz}$ before the averages were calculated. Both gain and phase have been expressed relativc to lincar acccleration. The phasc lags of $180^{\circ}\left(\mathbf{S}_{\max }\right)$ corrcspond to the vector distributions that are plotted relative to head position in Figure 10. The solid lines represent the fits of the transfer functions presented in Table 1. 


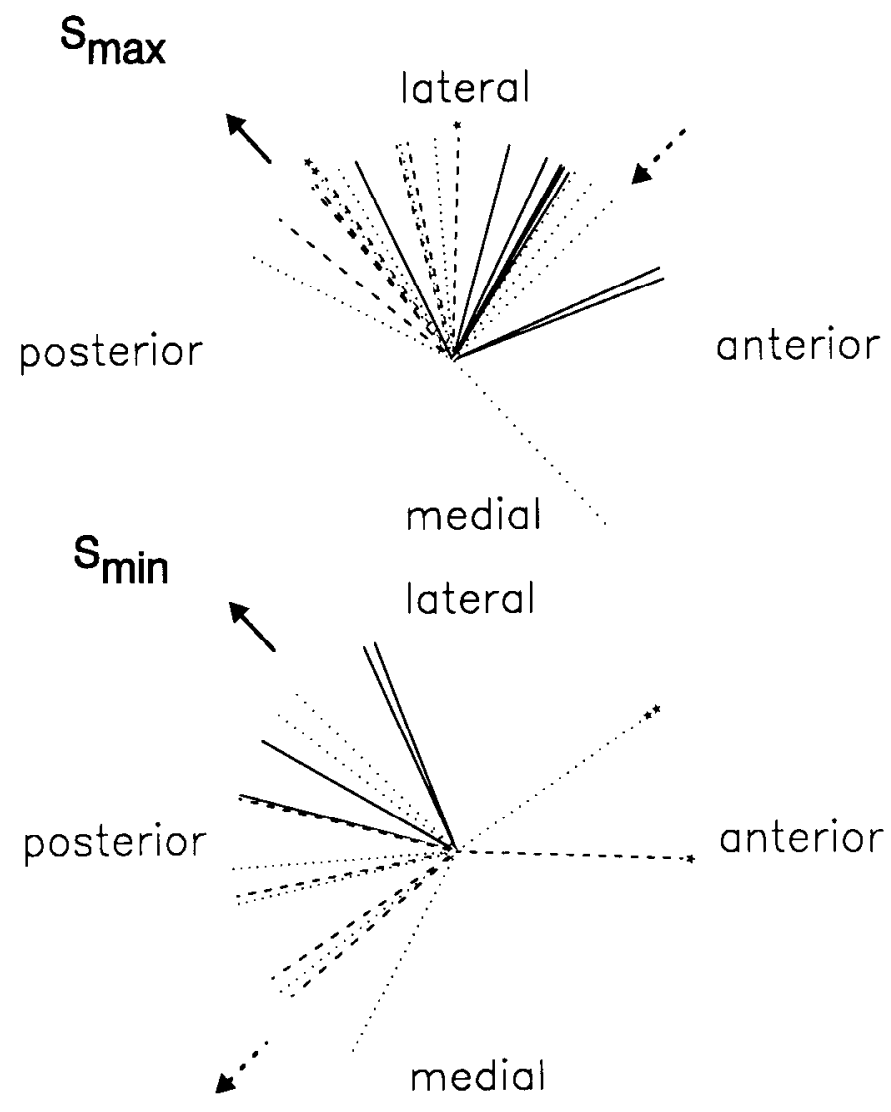

Figure 10. Neural response vector distributions for VC neurons (top, $\mathbf{S}_{\max } ;$ bottom, $\mathbf{S}_{\min }$ ). Vector directions are plotted relative to head position, in accordance with the phase plots of Figures 8 and 9. Cells tested at multiple frequencies are represented by their average direction. The vectors plotted have equal lengths and do not depict sensitivity differences among neurons. Solid lines are used for type I LA neurons $(n=$ 8). Dashed lines have been used for type I LP neurons $(n=7)$. Dotted lines have been used for the remaining VC neurons $(n=10)$. Only cells with tuning ratio greater than 0.1 have been included in the $\mathbf{S}_{\min }$ plot (bottom). The vectors of the two CW VC neurons have been marked with asterisks (the vectors with double asterisks belong to one of the marked neurons in Fig. 8). The LA and LP semicircular canal activation axes are indicated by the solid and broken arrows, respectively (Daunicht and Pellionisz, 1987). The arrows point toward the direction of rotation (linked by the right-hand rule) that causes excitation of the respective primary canal afferents.

done based on the hypothesis of a different functional role for the linear acceleration sensitivity of $\mathrm{HC}$ and $\mathrm{VC}$ neurons. If the linear acceleration sensitivity of $\mathrm{VC}$ neurons is appropriate for the generation of the vertical and torsional tilt LVORs, it should be compensatory to head position, not dynamic linear acceleration. $\mathrm{HC}$ neurons, on the other hand, should have responses that are compensatory to dynamic linear acceleration needed for the generation of horizontal translational LVOR.

Since the response vectors of VC neurons in Figure 10 have been plotted relative to head position, a clustering of $S_{\max }$ vectors in the ipsilateral (left) two quadrants indicates that these neurons should be excited with ipsilateral ear down. Examination of the top plot in Figure 10 indicates that seven of eight type I LA neurons (solid lines) had $\mathbf{S}_{\max }$ vectors in the anterolateral quadrant. Similarly, seven of seven type I LP neurons (dashed lines) had $\mathbf{S}_{\max }$ vectors in the posterolateral quadrant. The solid arrows show the VC sensitivity axes. The arrows point to the direction of rotation (linked by the right-hand rule) that excites the ipsilateral (left) anterior (solid arrow) and postcrior (broken arrow) canals. Therefore, the $\mathbf{S}_{\max }$ vectors of VC neurons were directed in such a way that the "canal" and "otolith" response of each cell would be "synergistic" during tilting of the head from the upright position. None of the VC cells in the present study that were tested for a type I/type II VC response $(n=15)$ were identified as type II LA or LP neurons. One of the remaining 10 VC neurons that were not identified according to their type I/type II VC response had the $\mathbf{S}_{\max }$ vector directed in the opposite direction compared to the rest of the cells (i.e., contralaterally). Based on the "synergy" between the canal and otolith responses exhibited by the rest of the cells, this neuron could have had a type II LP canal response.

The minimum sensitivity vectors of $\mathrm{VC}$ neurons are plotted in the bottom of Figure 10. Only 16 of $25 \mathrm{VC}$ cells exhibited significant two-dimensional sensitivity (i.e., tuning ratios larger than 0.1; see Materials and Methods) to allow identification of the direction of $\mathbf{S}_{\min }$. Unlike $\mathrm{HC}$ neurons in which the $\mathbf{S}_{\min }$ vectors were located either $\mathrm{CW}$ or $\mathrm{CCW}$ to $\mathrm{S}_{\max }$, only 2 of 16 VC neurons were CW (they are marked with asterisks in Fig. 10 ). Therefore, the distribution of the remaining $14 \mathbf{S}_{\min }$ vectors was similar to that of $\mathbf{S}_{\max }$ and rotated CCW by $90^{\circ}$ (Fig. 10). As seen from the figure, $\mathbf{S}_{\min }$ vectors were distributed around the respective canal sensitivity axes. That is, type I LA neurons had $\mathbf{S}_{\min }$ vectors clustered around the LA canal axis (solid arrow). Similarly, type I LP neurons had $\mathbf{S}_{\min }$ vectors closer to the LP canal axis (broken arrow).

\section{Discussion}

We have examined the spatial and temporal properties of neurons in the vicinity of the MVN in decerebrate rats during stimulation with pure linear acceleration in the horizontal head plane. Using a recently developed model to quantify the response properties of spatially tuned neurons (Angelaki, 1991a; Angelaki et al., 1992), we were able for the first time to describe the frequency dependence of the two-dimensional spatiotemporal response properties in otolith/canal-convergent vestibular nuclei neurons. We found that the response dynamics of otolith/canalconvergent vestibular nuclei neurons during stimulation with pure linear acceleration depended on the canal sensitivity of the cell. For example, $\mathrm{HC}$ neurons had maximum sensitivity vectors that reliably encoded linear acceleration at least up to $1 \mathrm{~Hz}$. In addition, when stimulated along their minimum sensitivity vector, $\mathrm{HC}$ neurons encoded the time derivative of linear acceleration (jerk). In contrast, the majority of VC neurons had small and relatively flat minimum sensitivity vector gains; however, they exhibited a strong gain decrease with increasing frequency when stimulated along their maximum sensitivity dircction.

Based on these results, the following three points will be addressed below. First, most otolith/canal (HC and VC)-convergent cells encountered in the MVN of decerebrate rats can be characterized by two-dimensional spatial and temporal sensitivity to linear acceleration. Second, the response properties to linear acceleration of $\mathrm{HC}$ and $\mathrm{VC}$ neurons are compatible with known characteristics of the translational and tilt LVORs. Third, the data support a hypothesis regarding a specific spatial relationship between the otolith and canal inputs in convergent vestibular nuclei neurons. 
1. Two-dimensional spatiotemporal response properties to linear acceleration

\section{Two-dimensional spatial sensitivity}

It has been theoretically shown that neurons without significant harmonic distortion in their response properties to dynamic linear acceleration can exhibit two-dimensional (planar) sensitivity (Angelaki, 1991a). Indeed, we have found that the majority of vestibular nuclei neurons exhibit two-dimensional spatial sensitivity at $0.6 \mathrm{~Hz}$ (Bush, Perachio, and Angelaki, unpublished observations). An interesting finding of the present study is the demonstration of the frequency dependence of the two-dimensional sensitivity. Both $\mathrm{HC}$ and VC cells (with the exceptions of the neurons marked with asterisks in Figs. 5, 8) showed an increasing two-dimensional sensitivity (quantified by the tuning ratio) at higher stimulus frequencies. The dependence of tuning ratio on frequency makes the separation of central vestibular cells into one- and two-dimensional linear accelerometer neurons difficult. In fact, neurons seem to manifest both one-dimensional and two-dimensional characteristics in different frequency bandwidths.

\section{Two-dimensional temporal sensitivity}

The theoretical formulation describing the response properties of neurons having two-dimensional spatial sensitivity with two response vectors in spatial and temporal quadrature (Angelaki, 1991a; Angelaki et al., 1992) does not require that one of the vectors be the time derivative of the other. For a derivative relationship to exist between $\mathbf{S}_{\max }$ and $\mathbf{S}_{\min }$, two requirements must be satisfied. First, the two vectors must be in temporal quadrature (i.e., have a phase difference of $90^{\circ}$ ) at each stimulus frequency. Second, the tuning ratio must change 10 -fold per decade of frequency. If the tuning ratio increases as a function of frequency, $\mathbf{S}_{\min }$ is the time derivative of $\mathbf{S}_{\max }$. Alternatively, if the tuning ratio decreases, $\mathbf{S}_{\max }$ is the derivative of $\mathbf{S}_{\min }$.

The first requirement for a derivative relationship between $\mathbf{S}_{\max }$ and $\mathbf{S}_{\min }$ is always satisfied in neurons with two-dimensional spatial sensitivity (Angelaki, 1991a). However, the second requirement is independent and does not follow as a consequence of the two-dimensional spatial sensitivity. The tuning ratio plots of Figure 4 indicate that the majority of the neurons examined here exhibited the appropriate frequency dependence of the tuning ratio to satisfy the second requirement for a derivative relationship between $\mathbf{S}_{\max }$ and $\mathbf{S}_{\min }$. We qualified this observation by fitting transfer functions to the frequency domain data for $\mathbf{S}_{\max }$ and $\mathbf{S}_{\min }$. A function describing the frequency dependence of $S_{\min }$ was the time derivative of that used to describe the frequency dependence of $S_{\max }$. Thus, for the majority of $\mathrm{HC}$ and VC neurons, the $\mathbf{S}_{\min }$ vector could be regarded as being proportional to the time derivative of $\mathbf{S}_{\max }$. A derivative relationship between $\mathbf{S}_{\max }$ and $\mathbf{S}_{\min }$ suggests that the responses of these neurons to linear acceleration are two-dimensional in terms of both space and time (frequency). Thus, the otolith/canal-convergent vestibular nuclei neurons identified in this study could be characterized as two-dimensional spatiotemporal linear accelerometers.

\section{Generation of the two-dimensional spatiotemporal response properties}

Two-dimensional spatial sensitivity can be generated from STC (Baker et al., 1984; Angelaki, 1992b). Convergence of units with different spatial and temporal characteristics creates target neu- rons with planar spatial properties (Angelaki, 1992b; Schor and Angelaki, 1992). However, two-dimensional temporal sensitivity is not a general propcrty of STC. That is, STC can crcatc spatial but not necessarily temporal two-dimensional sensitivity.

Primary otolith afferents exhibit a considerable variation in their spatial and temporal properties (Fernandez and Goldberg, 1976a-c; Goldberg et al., 1990a,b). Specifically, regular otolith afferents have relatively flat dynamics. Irregular otolith afferents have phasic or phasic-tonic characteristics, and their temporal dynamics have been described by a combination of fractional leaky differentiators and integrators (Goldberg et al., 1990a). Is STC between primary otolith afferents sufficient to explain the two-dimensional temporal properties in these neurons? A theoretical investigation of this question has shown that simple linear convergence between regular and irregular primary otolith afferents with different maximum sensitivity vector directions results in two-dimensional linear accelerometer neurons with $\mathbf{S}_{\max }$ encoding linear acceleration and $\mathbf{S}_{\min }$ transducing "jerk" (Angelaki, 1991b, 1992a, 1993). Thus, the two-dimensional spatiotemporal sensitivity to linear acceleration could be a direct consequence of the temporal dynamics of primary otolith afferents and their STC onto higher-order neurons.

\section{Comparison with previous studies}

The present study differs from previous similar studies (Melvill Jones and Milsum, 1969; Lannou et al., 1980; Perachio, 1981; Schor and Miller, 1982; Schor et al., 1984; Xerri et al., 1987) in two important ways. First, the linear acceleration sensitivity of the neurons was quantified using a two-dimensional treatment of their spatiotemporal response properties (Angelaki, 1991a; Angelaki et al., 1992). Second, our study concentrates on neurons that receive convergent otolith and canal inputs. These neurons were characterized in terms of their major canal input as $\mathrm{HC}$ or $\mathrm{VC}$ cells (prior to being tested with pure linear acceleration stimuli).

In response to linear acceleration stimuli, increasing (Schor, 1974; Schor and Miller, 1982; Schor et al., 1985), decreasing (Perachio, 1981; Xerri et al., 1987), as well as relatively flat response gains (Schor et al., 1984) with frequency have been observed. There are at least two possible reasons for the observed diversity of the frequency response characteristics of central otolith-sensitive neurons. First, the frequency dependence could vary with stimulus direction (Fig. 3). The response properties of central otolith neurons have often been studied along a single spatial orientation (Lannou et al., 1980; Schor and Miller, 1982; Xerri et al., 1987). Second, we have demonstrated here that an important variable in the study of the frequency response of the central otolith system is the characterization of the neurons in terms of their major canal input. It has been shown in the present study that $\mathrm{HC}$ and VC neurons exhibit different frequency dependencies of the $\mathbf{S}_{\max }$ and $\mathbf{S}_{\min }$ vectors. Neurons that receive only otolith input were not examined. It is possible that purely otolith cells (i.e., without convergent canal input) would have frequency response dynamics that are different from those reported here for $\mathrm{HC}$ and $\mathrm{VC}$ cells.

The described VC neurons had vectors that were spatially distributed similarly to those previously reported for cells in the lateral and descending vestibular nuclei of decerebrate cats (Schor et al., 1984): there was a lack of vectors in the pitch direction (anterior-posterior; Fig. 10) and a clustering of vectors close to 
the vertical semicircular canal planes. In addition, most neurons were excited by ipsilateral tilt, like the majority of the utricular primary afferents (Loe et al., 1973; Fernandez and Goldberg, 1976a; Tomko et al., 1981; Dickman et al., 1991). Unlike the VC neurons described here and the canal-unidentified neurons reported by Schor et al. (1984), HC cells had maximum sensitivity vectors that were spread throughout the horizontal plane. In addition, there was no direction predominance: some neurons would be excited during ipsilateral head tilt, whereas others would be excited during contralateral head tilt.

The variability in response dynamics of central otolith neurons and their dependence on stimulus orientation presents a difficulty to a quantitative evaluation of neuronal responses during combined angular and linear accelerations. For example, Kasper et al. (1988) and Wilson et al. (1990) studied the response dynamics of vestibular nuclei neurons during simultaneous stimulation with both angular and linear acceleration in canalintact animals. They separated neurons into otolith-, canal-, and otolith + canal-scnsitive cells on the basis of their responsc dynamics. Neurons that showed gain increases of threefold or more between 0.1 and $1 \mathrm{~Hz}$ and phase values larger than $55^{\circ}$ at 0.5 $\mathrm{Hz}$ were classified as canal (VC) cells (Kasper et al., 1988; Wilson et al., 1990). The results reported here demonstrate that neurons could exhibit these same response properties during stimulation with pure linear acceleration (i.e., along $\mathbf{S}_{\min }$ ).

\section{Potential relationship with otolith-ocular reflexes}

In the introductory remarks, it was noted that there were three types of otolith-ocular reflexes, each of them requiring signals with distinct spatial and temporal properties. The neuronal responses reported here have spatiotemporal properties in line with the requirements of these reflexes. In considering this issue, it should be emphasized that no attempt was made to identify our neurons as projecting to the ocular motor nuclei.

\section{HC neurons and horizontal translational LVOR}

During linear head translation in the horizontal plane, horizontal eye movements are elicited that are compensatory to head translation (Hess and Dieringer, 1991; Paige and Tomko, $1991 \mathrm{a}, \mathrm{b})$. The temporal properties of this reflex require that the system respond as a high-pass filter of linear acceleration (Niven et al., 1966; Mayne, 1974; Tokita et al., 1981; Paige and Tomko, 1991a). Accordingly, otolith/HC-convergent neurons should exhibit temporal and spatial response properties that are appropriate for the generation of the horizontal translational LVOR. If vestibular nuclei neurons responded as one-dimensional accelerometers, one would expect a restricted distribution and a high-pass filtered frequency response of their maximum sensitivity vectors. However, neither the spatial nor the temporal response properties of the maximum sensitivity vectors in our HC ncurons confirmed this expectation. The distribution of $\mathbf{S}_{\max }$ vectors was broad, extending throughout the horizontal head plane, while the frequency response for most neurons was flat without the high-frequency gain increase that governs the dynamics of the horizontal translational LVOR (Paige and Tomko, 1991a). However, along their minimum sensitivity vector, HC neurons have temporal and spatial characteristics compatible with the properties of the horizontal translational LVOR. For example, the frequency response of the $\mathbf{S}_{\min }$ vectors could provide the high-pass filtered linear acceleration signal necessary for generation of the horizontal translational LVORs. The tem- poral properties of $\mathbf{S}_{\min }$ showed an average phase lead of approximately $90^{\circ}$ relative to linear acceleration at the higher frequency range (Fig. 6). Neural responses that lead head acceleration by $90^{\circ}$ are $180^{\circ}$ out of phase with head velocity. Therefore, signals with temporal properties similar to those of $\mathbf{S}_{\min }$ could generate horizontal eye velocity that is compensatory (i.e., opposite in polarity) to linear head velocity. In part, the output of the $\mathrm{HC}$ neurons is further processed through the horizontal velocity to position integrator (Robinson, 1981).

\section{$V C$ neurons and tilt LVORs}

The second hypothesis addressed in this study relates to the spatial and temporal response properties of VC neurons. One category of otolith-ocular reflexes, the tilt LVORs, consists of vertical and torsional eye movements with low-pass filter characteristics during stimulation with linear acceleration in the horizontal head plane (Lichtenberg et al., 1982; Hess and Dieringer, 1991; Paige and Tomko, 1991a). If the temporal signals neccssary for the generation of the tilt LVORs were present in the vestibular nuclei, it would be expected that VC neurons would exhibit maximum sensitivity vectors with low-pass filter characteristics. Indeed, the responses of the majority of VC neurons ( 9 of 11) that were successfully tested at multiple orientations and frequencies were shown to have temporal properties that are consistent with a low-pass filter (Fig. 9). A comparison of the frequency response of $\mathbf{S}_{\max }$ dynamics in VC neurons and the dynamics of the vertical and torsional tilt LVORs in monkeys (Paige and Tomko, 1991a) and rats (Hess and Dieringer, 1991) suggests that the responses of these neurons could be appropriate for the generation of the tilt LVORs without being integrated. This is consistent with the persistence of the normal static counterroll reflexes in the cat following kainic acid lesions of the interstitial nucleus of Cajal (Anderson, 1981). The interstitial nucleus of Cajal has been shown to be a necessary structure for the normal function of the vertical and torsional velocity to position integrator (Fukushima, 1991).

Hess and Dieringer (1991) have proposed projections from hair cells in specific areas of the utricular macula to specific eye muscles. The alignment of the $\mathbf{S}_{\max }$ vectors of VC cells with the projection of the neuron's canal sensitivity plane (Fig. 10) supports this assumption and further suggests that these proposed utricular connections might, at least partly, be conveyed through otolith/canal-convergent neurons whose otolith and canal inputs are "synergistic" during normal movements of the head.

\section{Coordinate frame for otolith signals}

\section{Spatiotemporal properties in three dimensions}

Two-dimensional linear accelerometer neurons tested in three dimensions have spatiotemporal response properties that can also be described by two principal vectors in spatial and temporal quadrature (defining a "parent ellipse"; Angclaki, 1991a; Angelaki et al., 1992). The response ellipse determined from the neuronal responses during stimulation in the horizontal head plane represents the projection of the "parent ellipse" on the horizontal plane (Angelaki et al., 1992). As expected from true two-dimensional linear accelerometers tested in three dimensions, there is always a single direction in space, normal to the "parent ellipse" plane, along which the neurons exhibit zero sensitivity. The presence of the zero sensitivity direction suggests that, when testing of the neuronal responses is restricted to a single plane, the lack of a significant two-dimensional sen- 
sitivity does not necessarily imply that the cell has one-dimensional properties. Instead, it could simply mean that the stimulus plane may be perpendicular to that of the "parent ellipse" (i.e., it includes the zero sensitivity vector). Since the stimulus protocol in the present study did not include stimulation of the neurons along directions outside the horizontal head plane, we can make no definite statements regarding the orientation of the principal vectors and the properties of these cells in three dimensions. However, as will be discussed below, the specific relationship between the directions of the canal axis that constitutes the major input to the cell and the two response vectors to linear acceleration in the horizontal head plane supports the hypothesis that these observations made in a single plane underlie a more general organization and plane-specific otolith/ canal convergence in the central vestibular system.

\section{Plane-specific otolith/canal convergence}

A canal coordinate frame, common to all sensory inputs with spatial properties converging onto the vestibular nuclci, has becn previously suggested (Simpson et al., 1988; Hess and Dieringer, 1991; Soechting and Flanders, 1992). We would like to extend this suggestion to include two-dimensional linear accelerometer neurons (the latter are organized in response planes rather than response axes). Thus, plane-specific otolith/canal convergence might take place within the central vestibular system as follows. The plane of rotation for which the cell responds maximally (canal response) and the plane of the principal response vectors in three dimensions ("parent ellipse" plane for the otolith response) are parallel. Equivalently, the canal maximum activation direction (representing the axis of rotation for which the cell responds maximally; cf. Fukushima et al., 1990) and the zero sensitivity vector of the two-dimensional linear acceleration sensitivity of central vestibular neurons are in close alignment.

The distributions of linear acceleration response vectors for both $\mathrm{HC}$ and VC neurons determined in the present study are in agreement with the aforementioned hypothesis regarding a plane-specific otolith/canal convergence. For example, based on the hypothesis, HC neurons would have "parent ellipse" planes that are approximately parallel to the head horizontal plane. Within the horizontal plane, the maximum sensitivity vectors could be distributed throughout the plane (Fig. 7). VC neurons would have "parent ellipse" planes that are approximately parallel to the VC planes. Therefore, when their responses are examined in a plane perpendicular to their "parent ellipse" plane (i.e., horizontal head plane), the $\mathbf{S}_{\max }$ vectors are expected to cluster around the line of intersection of the two planes and the $\mathbf{S}_{\min }$ vectors to lie close to the respective canal axes. The VC vector distribution in Figure 10 supports this organization.

An additional consequence of the plane-specific otolith/canal convergence hypothesis is the prediction of small $\mathbf{S}_{\min }$ gains in VC neurons when tcsted in the horizontal head plane. Indeed, the majority of VC neurons encountered in the rat vestibular nuclei had small minimum sensitivity vectors. For example, all nine neurons in Figure 8 showing decreasing $\mathbf{S}_{\max }$ gain versus frequency have $S_{\min }$ gains that are less than $10 \mathrm{spikes} / \mathrm{sec} / g$ for all frequencies. Further, VC neurons had smaller $\mathbf{S}_{\text {min }}$ gains compared to $\mathrm{HC}$ cells. Thus, the lack of a significant $\mathbf{S}_{\min }$ sensitivity in VC neurons could be due to the zero sensitivity vector of these cells lying close to the horizontal head plane. The presence of a nonzero $\mathbf{S}_{\min }$ sensitivity in some VC neurons could arise because of the following reasons. First, the exact canal sensitivity plane was not determined in the present study. Second, the stimulus plane, which was defined as "head horizontal" and tilted from the stereotaxic horizontal by $26^{\circ}$, might not coincide with the plane perpendicular to the VC planes in all animals (Daunicht and Pellionisz, 1987; Blanks and Torigoe, 1989).

\section{Angular velocity sensitivity of the otolith system}

As discussed above, the demonstrated spatial and temporal properties of HC neurons with two-dimensional spatiotemporal sensitivity to linear acceleration identified in the MVN of rats could explain the spatial and temporal properties of the compensatory horizontal translational LVOR. However, two-dimensional spatiotemporal sensitivity to linear acceleration is not necessary for the generation of the horizontal translational LVOR. An alternate possibility could be that one-dimensional otolith afferent signals, which had been processed by a highpass filter and had specific connections to horizontal extraocular muscles (Hess and Dieringer, 1991), could generate the horizontal translational LVOR.

It is important to point out that two-dimensional sensitivity has additional significance for the otolith-ocular system. In addition to the translational and tilt LVORs, there is a third function of the otolith-ocular reflexes: the off-vertical axis VOR. During rotation about axes tilted from the earth-vertical, the otolith system demonstrates an angular velocity sensitivity (cf. Hess and Dieringer, 1990). How can a one-dimensional linear accelerometer function as a rate sensor? Although theoretical models have been previously prcposed that suggest how onedimensional linear accelerometers could estimate angular velocity (Hain, 1986; Raphan and Schnabolk, 1988; Hess, 1992), there are at present no neurophysiological data for their support. Alternatively, a two-dimensional spatiotemporal linear accelerometer is by definition a rate sensor (Angelaki, 1992c). The rate of change (derivative) of a rotating vector is proportional to the velocity of rotation:

$$
\mathbf{J}=-\mathbf{\Omega} \times \mathbf{G},
$$

where $\mathbf{J}$ is the "jerk" vector, $\mathbf{G}$ is the gravity vector, and $\boldsymbol{\Omega}$ is the angular frequency of rotation. Therefore, a two-dimensional spatiotemporal linear accelerometer neuron that encodes the two perpendicular vectors $\mathbf{J}$ and $\mathbf{G}$ carries information about the component of the three-dimensional angular velocity vector normal to the plane of $\mathbf{J}$ and $\mathbf{G}$. This information can be extracted by further processing (Angelaki, 1992d) and used to generate compensatory ocular responses during head rotation about non-earth-vertical axes. Based on the proposed threedimensional plane-specific otolith/canal convergence hypothesis, each otolith/canal-convergent neuron would carry information about an otolith-encoded component of the three-dimensional angular velocity vector that is parallel to its canal sensitivity axis. Thus, the two-dimensional spatiotemporal organization of the linear acceleration sensitivity in $\mathrm{HC}$ neurons could not only explain the generation of the horizontal translational LVOR, but also provide an easy and efficient way of transforming dynamic spatiotemporal linear acceleration into horizontal angular velocity sensitivity. The angular velocity sensitivity of the otolith system performs a function similar to that offered by the optokinetic system in the light: it complements the canal VOR in achieving ocular compensation over a frequency bandwidth approximating that of normal head movements. 


\section{References}

Anderson JH (1981) Ocular torsion in the cat after lesions of the interstitial nucleus of Cajal. Ann NY Acad Sci 545:865-871

Angelaki DE (1991a) Dynamic polarization vector of spatially tuned neurons. IEEE Trans Biomed Eng 38:1 1:1053-1060.

Angelaki DE (1991b) Spatio-temporal convergence in the otolith vestibular system. PhD thesis, University of Minnesota, Minneapolis.

Angclaki DE (1992a) Vcstibular neurons encoding two-dimensional linear acceleration assist in the estimation of rotational velocity during off-vertical axis rotation. Ann NY Acad Sci 656:910-913.

Angelaki DE (1992b) Spatio-temporal convergence (STC) in otolith neurons. Biol Cybern 67:83-96.

Angelaki DE (1992c) Two-dimensional coding of linear acceleration and the angular velocity sensitivity of the otolith system. Biol Cybern $67: 511-522$

Angelaki DE (1992d) Detection of rotating gravity signals. Biol Cybern 67:523-533.

Angelaki DE (1993) Spatial and temporal coding in single neurons. Biol Cybern, in press.

Angelaki DE, Bush GA, Perachio AA (1992) A model for the characterization of the spatial properties in vestibular neurons. Biol $\mathrm{Cy}$ bern 66:231-241.

Baarsma EA, Collewijn H (1975) Eye movements due to linear accelerations in the rabbit. J Physiol (Lond) 245:227-247.

Baker J, Goldberg J, Hermann G, Peterson B (1984) Spatial and temporal response properties of secondary neurons that receive convergent input in vestibular nuclei of alert cats. Brain Res 294:138-143.

Blanks RHI, Torigoe Y (1989) Orientation of the semicircular canals in rat. Brain Res 487:278-287.

Bush GA (1991) The spatial and temporal response properties of semicircular canal neurons of the medial vestibular nuclei responding to horizontal linear translation. $\mathrm{PhD}$ thesis, University of Texas Medical Branch, Galveston.

Bush GA, Perachio AA, Angelaki DE (1992) Quantification of different classes of canal-related vestibular nuclei neuron responses to linear acceleration. Ann NY $\Lambda$ cad Sci 656:917-919.

Correia MJ, Money KE (1970) The effect of blockage of all six semicircular canal ducts on nystagmus produced by dynamic linear acceleration in the cat. Acta Otolaryngol (Stockh) 69:7-16.

Curthoys IS, Markham CH (1971) Convergence of labyrinthine influences on units in the vestibular nuclei of the cat. I. Natural stimulation. Brain Res 35:469-490.

Daunicht WJ, Pellionisz AJ (1987) Spatial arrangement of the vestibular and the oculomotor system in the rat. Brain Res 435:48-56.

Dickman JD, Angelaki DE, Correia MJ (1991) Response properties of gerbil otolith afferents to small angle pitch and roll tilts. Brain Res 556:303-310.

Duensing F, Schaefer KP (1959) Uber die Konvergenz verschiedener labyrintharer Afferenzen auf einzelne Neurone des Vestibulariskerngebietes. Arch Psychiatr Nervenkr 199:345-371.

Estes MS, Blanks RHI, Markham CH (1975) Physiologic characteristics of vestibular first-order canal neurons in the cat. I. Response plane determination and resting discharge characteristics. J Neurophysiol 38:1232-1249.

Fernandez C, Goldberg JM (1976a) Physiology of peripheral neurons innervating otolith organs of the squirrel monkey. I. Response to static tilts and to long duration centrifugal force. J Neurophysiol 39:970984.

Fernandez C, Goldberg JM (1976b) Physiology of peripheral neurons innervating otolith organs of the squirrel monkey. II. Directional selectivity and force response relations. J Neurophysiol 39:985-995.

Fernandez C, Goldberg JM (1976c) Physiology of peripheral neurons innervating otolith organs of the squirrel monkey. III. Response dynamics. J Neurophysiol 39:996-1008.

Fukushima K (1991) The interstitial nucleus of Cajal in the midbrain reticular formation and vertical eye movement. Neurosci Res 10:159187.

Fukushima K, Perlmutter SI, Baker JF, Peterson BW (1990) Spatial properties of second-order vestibulo-ocular relay neurons in the alert cat. Exp Brain Res 81:462-478.

Goldberg JM, Fernandez C (1982) Eye movements and vestibular nerve responses produced in the squirrel monkey by rotations about an earth-horizontal axis. Exp Brain Res 46:393-402.
Goldberg JM, Desmadryl G, Baird RA, Fernandez C (1990a) The vestibular nerve of the chinchilla. IV. Discharge properties of utricular afferents. J Neurophysiol 63:781-790.

Goldberg JM, Desmadryl G, Baird RA, Fernandez C (1990b) The vestibular nerve of the chinchilla. $V$. Relation between afferent discharge properties and peripheral innervation patterns in the utricular macula. J Neurophysiol 63:791-804.

Hain TC (1986) A model of the nystagmus induced by off vertical axis rotation. Biol Cybern 54:337-350.

Hess BJM (1992) Three-dimensional head angular velocity detection from otolith afferent signals. Biol Cybern 67:323-333.

Hess BJM, Dieringer N (1990) Spatial organization of the maculoocular reflex of the rat: responses during off-vertical axis rotation. Eur J Neurosci 2:909-919.

Hess BJM, Dieringer N (1991) Spatial organization of linear vestibuloocular reflexes of the rat: responses during horizontal and vertical linear acceleration. J Neurophysiol 66:1805-1818.

Kasper J, Schor RH, Wilson VJ (1988) Response of vestibular neurons to head rotations in vertical planes. I. Response to vestibular stimulation. J Neurophysiol 60:1753-1764.

Lannou J, Cazin L, Hamann KF (1980) Responses of central vestibular neurons to horizontal linear acceleration in the rat. Pfluegers Arch 385:123-129.

Lichtenberg BK, Young LR, Arrott AP (1982) Human ocular counterrolling induced by varying linear accelerations. Exp Brain Res 48 127-136.

Loe PR, Tomko DL, Werner G (1973) The neural signal of angular head position in primary afferent vestibular nerve axons. J Physiol (Lond) 219:29-50.

Mayne R (1974) A systems concept of the vestibular organs. In: Handbook of sensory physiology, Vol VI/2, Vestibular system (Kornhuber HH, ed), pp 493-580. Berlin: Springer.

Melvill Jones G, Milsum JH (1969) Neural response of the vestibular system to translational acceleration. In: Supplement to conference on systems analysis approach to neurophysiological problems, pp 8-20. Brainerd, $\mathbf{M N}$.

Niven JI, Hixson WC, Correia MJ (1966) Elicitation of horizontal nystagmus by periodic linear acceleration. Acta Otolaryngol (Stockh) 62:429-441.

Paige GD, Tomko DL (1991a) Eye movement responses to linear head motion in the squirrel monkey. I. Basic characteristics. J Neurophysiol 65:1170-1182.

Paige GD, Tomko DL (1991b) Eye movement responses to linear head motion in the squirrel monkey. II. Visual-vestibular interactions and kinematic considerations. J Neurophysiol 65:1183-1196.

Perachio AA (1981) Responses of neurons in the vestibular nuclei of awake squirrel monkeys during linear acceleration. In: The vestibular system: function and morphology (Gualtierotti T, ed), pp 443-451. New York: Springer.

Perachio AA, Dickman JD, Correia MJ (1988) Morphological and functional characteristics of semicircular canal afferents sensitive to head tilt. In: Basic and applied aspects of vestibular function (Hwang JC, Daunton NG, Wilson VJ, eds), pp 13-25. Hong Kong: Hong Kong UP.

Perachio AA, Bush GA, Angelaki DE (1992) A model of responses of horizontal canal-related vestibular nuclei neurons that respond to linear acceleration. Ann NY Acad Sci 656:795-801.

Raphan T, Schnabolk C (1988) Modeling slow phase velocity generation during off-vertical axis rotation. Ann NY Acad Sci 545:29-50.

Robinson DA (1981) The use of control systems analysis in the neurophysiology of eye movements. Annu Rev Neurosci 4:463-503.

Schor RH (1974) Responses of cat vestibular neurons to sinusoidal roll tilt. Exp Brain Res 20:347-362.

Schor RH, Angelaki DE (1992) The algebra of neural response vectors. Ann NY Acad Sci 656:190-204.

Schor RH, Miller AD (1982) Relationship of cat vestibular neurons to otolith-spinal reflexcs. Exp Brain Res 47:137-144.

Schor RH, Miller AD, Tomko DL (1984) Responses to head tilt in cat central vestibular neurons. I. Direction of maximum sensitivity. J Neurophysiol 51:136-146.

Schor RH, Miller AD, Timerick JB, Tomko DL (1985) Responses to head tilt in cat central vestibular neurons. II. Frequency dependance of neural response vectors. J Neurophysiol 53:1444-1452. 
Searles EJ, Barnes CD (1977) Ipsilateral utricular and semicircular canal interactions from electrical stimulation of individual vestibular nerve branches recorded in the descending medial longitudinal fasciculus. Brain Res 125:23-36.

Simpson JI, Leonard CS, Soodak RE (1988) The accessory optic system of rabbit. II. Spatial organization of direction of sclectivity. $J$ Neurophysiol 60:2055-2072.

Soechting JF, Flanders M (1992) Moving in three-dimensional space: frames of reference, vectors, and coordinate systems. Annu Rev Neurosci 15:167-191.

Tokita T, Miyata Han H, Masaki M, Ikeda S (1981) Dynamic characteristics of the otolithic oculomotor system. Ann NY Acad Sci 374: $56-68$.
Tomko DL, Peterka RJ, Schor RH (1981) Responses to head tilt in cat eight nerve afferents. Exp Brain Res 41:216-221.

Wilson VJ, Yamagata Y, Yates BJ, Schor RH, Nonaka S (1990) Response of vestibular neurons to head rotation in vertical planes. III. Response of vestibulocollic neurons to vestibular and neck stimulation. J Ncurophysiol 64:1695-1703.

Xerri C, Barthelemy F, Harlay L, Borel L, Lacour M (1987) Neuronal coding of linear motion in the vestibular nuclei of the alert cat. I. Response characteristics to vertical otolith stimulation. Exp Brain Res 65:569-581. 